\title{
LE ELEZIONI REGIONALI IN SICILIA DEL 5
} NOVEMBRE 2017

\author{
di Maurizio Cerruto e Marco La Bella*
}


Abstract - This article examines the outcome of the regional election held in Sicily on November 5, 2017. It was a victory for the center-right, which returns to power in the Region after the problematic term of the center-left president incumbent Rosario Crocetta. The center-right winner, Nello Musumeci, backed by a wide coalition but more right-oriented than in the past, has to be explained, on the one hand, with the low turnout - abstension reaching $53 \%$ - and, on the other hand, with the consolidation of the anti-establishment and protest movement Five Stars Movement (M5S) which in 2017 increased its consensus of 12 percentage points to 2012 and remained the single biggest party in the Sicily's assembly. The election results show a polarization both in the government of the Region and in the opposition and a strong personalization of the electoral competition - this latter being a typical aspect of the elections in Sicily, whose evidence concerning this election's candidates is discussed in the article.

Keywords: Participation, competition, Five Star Movement, polarization, personalization

*Per quanto l'articolo sia frutto di un'idea e di un lavoro comune si possono attribuire a Marco La Bella i paragrafi 1, 4 e 7, a Maurizio Cerruto $i$ paragrafi 2, 3, 5 e 6 . 


\section{La difficile presidenza Crocetta}

La XVI legislatura consegna agli elettori siciliani chiamati a votare il 5 novembre 2017 per l'elezione del Presidente della Regione e per il rinnovo dell'Assemblea Regionale Siciliana (Ars) una situazione politica molto delicata nella quale si ripropongono (quasi paradossalmente) alcuni temi ed alcune questioni a cui i governi presieduti da Rosario Crocetta non sono stati in grado di trovare soluzione, nonostante le aspettative e i buoni propositi.

Eletto Presidente della Regione siciliana il 28 ottobre 2012, con il 30,5\% dei consensi, Rosario Crocetta si è trovato ad affrontare una situazione politica inedita su cui hanno influito alcuni fattori significativi. Il primo fattore è rappresentato dal livello di astensionismo che ha raggiunto la soglia del 52,6\% - con una percentuale della partecipazione elettorale (46,7\%) nemmeno paragonabile a quella delle tornate elettorali che hanno portato all'elezione dei suoi predecessori: Raffaele Lombardo nel 2008 (66,7\%) e Salvatore Cuffaro nel 2006 $(59,2 \%)$ e nel $2001(63,5 \%)$ - e che ha rischiato di ridimensionare il valore dell'affermazione elettorale. Al dato sulla partecipazione va aggiunto il calo dei consensi elettorali ai partiti della coalizione di centrosinistra - a cui ha fatto da argine l'alleanza organica con l'Udc che non gli ha comunque consentito di ottenere la maggioranza dei seggi all'Ars - fermandosi a 39 seggi su 90. Il secondo fattore è rappresentato dal successo elettorale del M5s che ha favorito un significativo ricambio all'Ars, con l'avvenuta elezione di 15 deputati, contribuendo indirettamente all'alternanza del centro-sinistra con il centrodestra al governo della Regione. Il comportamento politico dell'Udc orientato a sviluppare un'alleanza organica con il Partito Democratico e il successo elettorale del M5s contribuiscono alla sperimentazione anche in Sicilia di un assetto politico "tripolare". Un assetto politico che sostanzialmente si regge sull'ampliamento della coalizione di centro-sinistra, da un lato, e sulla performance elettorale del M5s in grado di arginare la tradizionale capacità di aggregazione del consenso dei partiti di centro-destra, nonché di ridimensionare la tendenza all'astensionismo, dall'altro lato.

Ma vi è di più. Il risultato elettorale del 2012 va inquadrato anche nell'ambito della grave crisi economico-finanziaria della Regione siciliana che ha eroso quelle risorse pubbliche che, negli ultimi decenni, costituivano le principali armi per la conquista del consenso da parte del centrodestra. Una condizione questa che aveva condizionato i quattro anni di governo della presidenza Lombardo (predecessore di Crocetta), prima delle sue dimissioni (anticipate), segnati da crisi di governo latenti a cui si sono accompagnate numerosi avvicendamenti nella composizione della giunta e nella maggioranza di riferimento (Colloca e Vignati, 2013).

In questo mutato scenario politico notevoli erano le aspettative su un cambiamento di rotta sia per il profilo politico del presidente sia per la prima volta ${ }^{1}$ - dall'introduzione dell'elezione diretta del presidente - del centrosinistra al governo della Regione siciliana. Rosario Crocetta, ex sindaco di Gela (nonché eurodeputato del Pd), ha costruito la sua carriera politica intorno al suo impegno nella lotta alla mafia e nell'azione amministrativa improntata al rinnovamento - tanto da suggerire la definizione di «rivoluzione Sicilia».

Il Governo Crocetta della XVI legislatura pur nascendo sotto buoni auspici, però, si è trovato a fronteggiare una serie di condizionamenti politici interni alla coalizione che si aggiungono ai condizionamenti esterni di carattere economico-finanziario con il rigore e il controllo del deficit richiesto dal governo centrale al fine di limitare gli effetti della crisi economica.

Quanto ai condizionamenti interni essi sono figli del risultato elettorale che non ha garantito al governatore Crocetta una maggioranza sufficiente all'Ars. Questa condizione di non auto-

${ }^{1}$ La XII legislatura (1996-2001), in realtà, è stata la prima vera volta di un esponente dei Ds, Angelo Capodicasa, alla guida del governo della Regione siciliana e di una coalizione ampia di riferimento dell'area di centro-sinistra. L'esperienza ha avuto una durata relativamente breve, meno di due anni, dal 21/11/1998 al 25/07/2000. 
sufficienza era aggravata dalle divisioni interne al Pd siciliano che già in fase pre-elettorale aveva mostrato molti limiti non essendo stato capace di coagularsi attorno ad un leader prima dell'autocandidatura di Crocetta. A poco è servito l'effetto traino della leadership di Crocetta, dimostrata dal risultato elettorale sulla coalizione di centrosinistra, a cui si aggiungono le vicende politiche che hanno attraversato l'Udc - azionista di maggioranza della coalizione che nonostante il forte radicamento in Sicilia si è trovato in una condizione di difficoltà e di logoramento come sarà successivamente evidenziato dalle elezioni politiche del $2013 \mathrm{e}$ dall'uscita dalla giunta Crocetta nel settembre 2016 (Azzolina, 2013).

Il resto è (quasi) attualità. Tutto inizia con le difficoltà nella formazione della prima giunta dal profilo prevalentemente tecnico - per espressa volontà di Crocetta - con l'esclusione dei deputati anche tra quelli indicati dai partiti della coalizione di riferimento. La redistribuzione dei posti in giunta prevedeva l'indicazione di 4 assessori con una forte caratterizzazione tecnica di riferimento dell'area del Pd, 3 assessori indicati dall'Udc, 1 assessore proveniente dal mondo degli industriali (e di fiducia del presidente) e 4 assessori (esterni) per la cui scelta Crocetta ricorre a personalità in grado di imprimere un forte impatto nell'opinione pubblica e sull'immagine complessiva del governo siciliano ${ }^{2}-$ si veda Tab. 1.

Tab. 1 - Composizione partitica e durata delle giunte regionali in Sicilia nella XVI legislatura (20122017)

\begin{tabular}{|c|c|c|c|c|}
\hline & $\begin{array}{c}\text { Data } \\
\text { nomina }\end{array}$ & $\begin{array}{l}\text { Pres. } \\
\text { giunta }\end{array}$ & Part. Pres. & $\begin{array}{l}\text { Numero dei } \\
\text { componenti della } \\
\text { giunta per partito }\end{array}$ \\
\hline $\begin{array}{l}\text { Primo } \\
\text { Crocetta }\end{array}$ & $19 / 11 / 2012$ & Crocetta & $\begin{array}{l}\text { Il Mega- } \\
\text { fono }\end{array}$ & $\begin{array}{c}\text { Area Pd (4); } \\
\text { Tecnici area } \\
\text { Megafono (1) } \\
\text { Esterni (4); } \\
\text { Udc (3) }\end{array}$ \\
\hline $\begin{array}{l}\text { Crocetta } \\
\text { bis }\end{array}$ & $14 / 04 / 2014$ & Crocetta & $\mathrm{Pd}$ & $\begin{array}{l}\text { Area Pd (4); } \\
\text { Tecnici area } \\
\text { Megafono (5) } \\
\text { Esterni (1); } \\
\text { Udc (1); Arti- } \\
\text { colo } 4 \text { (1) }\end{array}$ \\
\hline $\begin{array}{l}\text { Crocetta } \\
\text { ter }\end{array}$ & $4 / 11 / 2014$ & Crocetta & $\mathrm{Pd}$ & $\begin{array}{l}\text { Area Pd (3); } \\
\text { Tecnici area } \\
\text { Megafono (3) } \\
\text { Esterni (1); } \\
\text { Udc (2); Inte- } \\
\text { rim (3) }\end{array}$ \\
\hline $\begin{array}{l}\text { Crocetta } \\
\text { quater }\end{array}$ & $4 / 11 / 2015$ & Crocetta & $\mathrm{Pd}$ & $\begin{array}{c}\text { Area Pd (6); } \\
\text { Tecnici area } \\
\text { Megafono (1) } \\
\text { Udc (2); } \\
\text { Sicilia Futura } \\
\text { (1); Ncd (1); } \\
\text { Interim (1) }\end{array}$ \\
\hline
\end{tabular}

L'approccio utilizzato da Crocetta nella nomina della giunta non ha riscosso grandi consensi nella coalizione soprattutto nel partito di maggioranza relativa $(\mathrm{Pd})$ il quale lamentava il ridimensionamento dei propri spazi di autonomia circa la possibilità di indicare delle figure in grado di rappresentare tutte le anime del partito democratico siciliano. Ciò nonostante, il primo periodo della legislatura è stato caratterizzato dal profilo tecnico della giunta regionale malgrado le pressioni dei partiti della coalizione e i primi avvicendamenti in giunta dovuti alle dimissioni di alcuni suoi componenti. A supporto della linea del presidente Crocetta è venuto, nelle primissime fasi, il sostegno dei deputati del M5s sotto forma di appoggio ester-

${ }^{2}$ I nomi di prestigio inseriti in giunta sono quelli di Lucia Borsellino - figlia di Paolo magistrato ucciso dalla mafia in via D'Amelio a Palermo nel 1992 - già dirigente dell'assessorato alla salute della Regione siciliana; Nicolò Marino ex pubblico ministero della Direzione Distrettuale Antimafia di Caltanissetta; Franco Battiato cantautore siciliano di fama internazionale molto impegnato anche sui temi politici e sociali; Antonino Zichichi fisico e divulgatore scientifico italiano, personalità prestigiosa a livello internazionale, fondatore del Centro di Cultura Scientifica Ettore Majorana di Erice. 
no in alcuni passaggi d'aula sulla base della condivisione di alcuni obiettivi del governo, il cosiddetto "modello Sicilia".

Questa condivisione porta, tra l'altro, all'approvazione della legge regionale n. 7 del 2013 sull'abolizione delle province. Una norma di indirizzo - composta da un solo articolo e tre commi - che rimandava ad una disciplina organica ma che (di fatto) apriva al processo di traghettamento dalle province regionali ai liberi consorzi comunali previsti dallo statuto siciliano ${ }^{3}$. Si è trattato di una legge "manifesto" nell'indirizzo della riduzione dei costi della politica più che di un intervento pubblico in grado di riformare l'assetto istituzionale locale, come dimostra l'evoluzione legislativa sul settore degli enti intermedi di area vasta in Sicilia. $\mathrm{Al}$ di là degli esperimenti politici, sul tavolo rimanevano alcune delle questioni politiche su cui si sarebbe dovuto misurare il governo Crocetta durante tutta la legislatura: il risanamento economico-finanziario a cui si ricollega il tema della trasparenza del bilancio e della riduzione della spesa; la riforma del settore della formazione professionale uno dei settori di maggiore espansione della spesa pubblica in Sicilia ${ }^{4}$; la riduzione delle società partecipate, proseguendo nel lavoro iniziato dal governo precedente di controllo della spesa pubblica e dei bilanci degli enti locali; infine, ma non ultimo, gli interventi nella sanità - settore strategico del bilancio della Regione - dove, dopo l'approvazione del piano di rientro del governo Lombardo, si rendeva necessario intervenire sulla rete ospedaliera.

Ben presto però il clima di collaborazione con il M5s perde la sua efficacia, e con lui il cosiddetto "modello Sicilia". Due questioni su tutte incrinano il clima di collaborazione: le elezioni politiche alle porte e la spinta alle larghe intese, e l'ingresso in maggioranza di altri due gruppi presenti all'Ars (Articolo 4 e, successivamente, Sicilia Futura).

Il risultato politico è sotto gli occhi di tutti e caratterizzerà l'esperienza del governo Crocetta della XVI legislatura. Una legislatura segnata da una condizione di stallo e di immobilismo politico che negli anni ha prodotto (quasi esclusivamente) un avvicendamento nelle compagini di governo e negli assessorati. Dalla nomina del primo governo Crocetta del 2012 è possibile contare almeno tre rimpasti di governo che di fatto hanno visto modificata strutturalmente la composizione della maggioranza di governo in relazione ai mutamenti del quadro politico regionale e nazionale.

Il primo rimpasto è quello del 14 aprile 2014, Crocetta-bis, che conclude una fase politica molto travagliata che ha caratterizzato il primo anno di vita della giunta Crocetta. Tra i fatti salienti del primo anno di vita del governo di centrosinistra la sostituzione dell'assessore all'economia Francesca D'Amelio a soli 5 giorni dalla nomina, così come la sostituzione di due delle quattro personalità esterne fortemente volute in giunta da Crocetta: Antonino Zichichi e Franco Battiato (ad aprile 2013). Il terzo componente esterno in giunta Nicolò Marino sarà sostituito con la nomina del Crocetta bis.

Al di là delle singole defezioni, le motivazioni politiche di questo primo rimpasto vanno ricercate nelle vicende politiche interne al partito democratico siciliano ${ }^{5}$. Anche il secondo rimpasto del novembre 2014, Crocetta-ter, a pochi mesi dal precedente, può essere inquadrato nell'ambito del dibattito interno al Pd siciliano e nazionale e ai nuovi equilibri tra le diverse anime del partito. In questo caso è l'area del partito che faceva riferimento a Gianni $\mathrm{Cu}-$ perlo a chiedere riconoscimento e spazio in giunta. Questa nuova fase è stata caratterizzata,

\footnotetext{
${ }^{3}$ Nello specifico, questa norma non conteneva indicazioni sulla governance e sulle funzioni dei nuovi enti di area vasta ma chiarisce che si tratterà di enti intermedi i cui vertici saranno eletti attraverso elezioni di secondo grado.

${ }^{4}$ Precedentemente il governatore Lombardo aveva cercato di riformare il settore senza successo. Il governo Crocetta è intervenuto prima con la rotazione dei dirigenti di questo settore come di altri. L'obiettivo era quello di fare partire un nuovo ciclo della formazione professionale attraverso l'introduzione di nuovi criteri per un "piano giovani".

${ }^{5}$ Ciò nonostante il Pd mantiene la stessa consistenza numerica nel nuovo governo regionale ma, nell'attribuzione delle deleghe ai nuovi assessori, viene premiata (quasi esclusivamente) l'area renziana dei Democratici riformisti. Il riposizionamento di Crocetta, inoltre, fa sì che egli stesso aderisca al gruppo del Pd, dopo il chiarimento sul doppio tesseramento che ha coinvolto la segreteria nazionale del Pd. Inoltre, viene ridimensionato il ruolo dell'Udc a vantaggio del gruppo politico di Articolo 4 che entra a far parte della maggioranza.
} 
ancora una volta, da una serie di dimissioni e nomine che hanno preceduto e seguito la presentazione della nuova giunta, come dimostrato dal ricorso frequente all'interim.

Ed ancora a scadenza annuale, nel novembre 2015, un rimpasto nella giunta di governo ha portato alla nascita del Crocetta-quater. A scatenare le turbolenze sul governo regionale sono state le indagini della magistratura relative all'affaire delle cliniche private e allo scandalo "Tutino", che ha portato alle dimissioni dell'assessore alla salute Lucia Borsellino. La giunta regionale che viene fuori da questa fase di crisi presenta una fisionomia diametralmente opposta a quella del primo governo Crocetta. Un rinnovato ruolo dei partiti che entrano in giunta con personalità forti del $\mathrm{Pd}$, come il capogruppo Gucciardi e il Presidente della Commissione Affari Istituzionali Cracolici, a cui si sono adeguati anche gli altri partiti della coalizione. Ad uscirne ridimensionato è il ruolo dei tecnici e degli esperti a cui ha fatto da contraltare il riconoscimento in giunta dell'appoggio dei gruppi parlamentari venuti a sostegno della maggioranza: Sicilia Futura e Ncd.

La parte finale della legislatura, infine, è segnata dall'uscita dalla maggioranza di governo dell'Udc. Essa costituisce il fatto politico più rilevante dopo la scelta coraggiosa del 2012 quando si era collocata organicamente nel raggruppamento di centrosinistra a sostegno di Crocetta, rappresentandone (forse) la principale novità politica. L'uscita dalla maggioranza per l'Udc non è stato un passaggio indolore. Le cronache politiche di quelle settimane, tra la fine del mese di settembre e il mese di ottobre del 2016, registrarono un duro scontro interno al partito tra l'ala governista - costituita da Giampiero D'Alia reggente del partito in Sicilia e i suoi più fedeli collaboratori, tra cui gli assessori in carica Giovanni Pistorio e Gianluca Miccichè - e il responsabile dell'Udc a livello nazionale Lorenzo Cesa, in quei giorni in Sicilia per riprendersi il partito, secondo alcuni addetti ai lavori. Per quest'ultimo l'obiettivo principale era quello di riposizionare il partito nell'ambito del centrodestra, in perfetta coerenza con la linea politica nazionale, ponendo così fine all'esperienza deludente nella maggioranza di centrosinistra a sostegno del presidente Crocetta.

Di lì in avanti ancora rimpasti più o meno significativi nella giunta di governo, confermando un po' la tendenza che ha caratterizzato l'intera legislatura. Alla scadenza naturale della XVI legislatura si contano 59 cambi di assessore, l'ultimo dei quali a soli 52 giorni dalle elezioni per il rinnovo del Parlamento siciliano. A conferma ulteriore della difficile presidenza Crocetta, inoltre, il dato sulle mozioni di sfiducia presentate e discusse in aula, ben tre. Nessuna di queste è andata a buon fine ma hanno evidenziato, qualora fosse necessario, il profondo malessere che ha attraversato tutte le fasi della prima esperienza del centrosinistra alla guida della Regione siciliana.

Sin qui le principali vicende politiche che hanno caratterizzato la XVI legislatura dell'Assemblea Regionale Siciliana. Una ricostruzione che costituisce una utile premessa all'analisi delle vicende politico-elettorali che hanno caratterizzato le elezioni regionali in Sicilia del 5 novembre 2017. Alla base della sonora sconfitta del centro-sinistra, solo in parte riconducibile alle divisioni interne alla coalizione e alla presentazione di due candidati d'area (Fabrizio Micari e Claudio Fava), ci sono proprio le scarse performance del governo uscente. Sono queste alcune delle questioni che approfondiremo, a partire delle "nuove" regole del gioco, il "nuovo" Statuto che riduce il numero dei deputati da 90 a 70 e la legge elettorale regionale (la legge regionale n. 7 del 2005), un sistema misto, proporzionale ma con un cor-

\footnotetext{
${ }^{6} \mathrm{Nel}$ giugno del 2015 è stato arrestato a Palermo il chirurgo plastico Matteo Tutino, ex primario di Villa Sofia, insieme ad altri ex manager dell'ospedale palermitano con le accuse di falso, abuso d'ufficio, truffa e peculato. A Matteo Tutino, medico personale del governatore Crocetta, e agli altri manager veniva contestato (tra l'altro) il fatto di avere ottenuto il rimborso da parte del servizio sanitario regionale di diverse operazioni private eseguite dal chirurgo. La vicenda ha avuto una forte rilevanza nazionale anche grazie alle rivelazioni dei giornalisti Messina e Zoppi che hanno pubblicato una presunta intercettazione telefonica tra Tutino e Crocetta nella quale il medico riferiva al governatore che l'assessore «Borsellino andava fermata, fatta fuori come suo padre». Le conseguenze politiche immediate sono state le dimissioni dell'assessore regionale alla salute Lucia Borsellino e l'autosospensione del Presidente Crocetta. Le indagini, successivamente, dimostrarono che le dichiarazioni pubblicate dai due giornalisti non erano sostenute da prove e quindi Crocetta è stato riabilitato dopo qualche settimana, mentre è ancora in corso il procedimento giudiziario contro i manager della sanità siciliana.
} 
rettivo maggioritario. Successivamente, ci soffermeremo ad analizzare l'offerta politica e la struttura della competizione, sia sotto il profilo delle liste regionali e dei rispettivi candidati alla presidenza, evidenziando temi e strategie della campagna elettorale, sia sotto il profilo dell'adeguamento di partiti e movimenti agli incentivi offerti dalla legge elettorale. Analizzeremo, quindi, i risultati elettorali - tanto sul versante della partecipazione al voto quanto su quello del voto ai partiti -, ed una delle costanti della storia elettorale siciliana, ovvero la personalizzazione del rapporto di rappresentanza. Infine, prima delle considerazioni conclusive, daremo conto della composizione della nuova Assemblea regionale siciliana e del nuovo governo presieduto da Nello Musumeci.

\section{Il "nuovo" Statuto e la legge elettorale regionale}

Le elezioni regionali del 2012 sono state le ultime che hanno portato alla elezione di 90 deputati all'Assemblea Regionale Siciliana; la prima novità delle elezioni del 5 novembre 2017 riguarda, infatti, il numero dei deputati. Ancor prima della norma sulla riduzione del numero dei consiglieri nelle regioni a statuto ordinario (ai sensi dell'art. 2 del d.1. 10 ottobre 2012, n. 174 convertito con modificazioni dalla legge 7 dicembre 2012, n. 213) e della relativa pronuncia della Corte Costituzionale (sentenza n. 198 del 2012, secondo la quale quelle regole erano applicabili solo alle regioni a statuto ordinario ma non alle regioni a statuto speciale), la Sicilia - assieme a Friuli-Venezia Giulia e Sardegna - aveva iniziato un iter di autoriduzione del numero di seggi. La norma era stata approvata dall'Ars già nel dicembre 2011 (XV legislatura Ars), ma per essere operativa la modifica statutaria richiedeva la doppia lettura di Camera e Senato, come previsto per le modifiche costituzionali. La Camera dei deputati ha dato il via libera definitivo al provvedimento che modifica gli statuti di Friuli-Venezia Giulia, Sicilia e Sardegna con le leggi costituzionali nn. 1, 2 e 3 del 7 febbraio 2013.

Grazie alla legge costituzionale n. 2 del 2013 i seggi all'Ars passano da 90 a 70 (ma ne basterebbero, secondo i calcoli del d.1. 138 del 2011 - poi conv. in 1.148 del 2011 - in base alla popolazione residente, cinquanta). Si ridisegna, così, la distribuzione dei seggi per l'elezione nelle nove province. Saranno, infatti, 62 (e non più 80 ) i deputati eletti con il sistema proporzionale: la provincia con il maggior numero di deputati eletti rimane Palermo con 16 seggi (prima erano 20), a Catania ne spettano 13 (prima 17), a Messina 8 (prima 11), ad Agrigento 6 (prima 7), a Siracusa e a Trapani 5 (prima, rispettivamente, 6 e 7), a Ragusa 4 (prima 5), a Caltanissetta 3 (prima 4), ad Enna 2 (prima 3) 7 . Seggi che vengono assegnati con il metodo del quoziente Hare e l'attribuzione dei più alti resti (con recupero sempre a livello provinciale) alle liste che abbiano superato la soglia del $5 \%$ a livello regionale.

A disciplinare in maniera organica la materia elettorale in Sicilia rimane, invece, la legge regionale n. 7 del 3 giugno 2005 ("Norme per l'elezione del Presidente della Regione siciliana a suffragio universale e diretto e nuove norme per l'elezione dell'Assemblea Regionale Siciliana") - approvata durante la presidenza di Salvatore Cuffaro alla Regione siciliana - che ha modificato in profondità l'impianto ereditato dalla lontana legge 20 marzo 1951, n. 29 (e successive modifiche) ${ }^{8}$, e sostituito la disciplina transitoria dettata in sede di revisione statutaria nel $2001^{9}$.

\footnotetext{
${ }^{7}$ La nuova distribuzione dei seggi è stata calcolata dividendo la cifra della popolazione residente in ciascuna circoscrizione provinciale per il quoziente fisso 80.692, ottenuto dividendo per 62 la cifra della popolazione legale residente nella regione all'ultimo censimento ufficiale fatto nel 2011.

${ }^{8}$ I deputati regionali venivano eletti in base "al sistema proporzionale puro stabilito dal d.1.lgt. 10 marzo 1946, n. 47”, cioè dalla legge elettorale utilizzata per la elezione della Assemblea Costituente, ed escludendo espressamente la possibilità di collegamento fra liste. La regione era ripartita in nove circoscrizioni elettorali corrispondenti alle province ed ogni elettore disponeva di un voto di lista e della possibilità di esprimere (a partire dal 1992) un'unica preferenza. I seggi venivano poi ripartiti con il quoziente naturale e i seggi che rimanevano da assegnare attribuiti in sede circoscrizionale alle liste con i più alti resti, comprese le liste che non avevano raggiunto alcun quoziente intero.

${ }^{9}$ In particolare, la legge costituzionale 31 gennaio 2001, n. 2 ("Disposizioni concernenti l'elezione diretta dei Presidenti delle Regioni a Statuto speciale e delle Province autonome di Trento e Bolzano") replica i medesimi meccanismi di elezione del presidente e di sfiducia "distruttiva" previsti per le Re-
} 
Anche in Sicilia, viene consolidata la forma di governo neoparlamentare (Vassallo e Baldini 2000; Ceccanti 2002) prevista dalla legge costituzionale n. 2 del 2001 e già trasposta nel testo statutario, confermando, da un lato, il principio secondo cui "il Presidente della Regione è eletto a suffragio universale e diretto, contestualmente all'elezione dell'Assemblea regionale" (art. 9, Statuto speciale) e, dall'altro, la formula proporzionale "corretta", ovvero un sistema misto, in larga parte proporzionale ma con un correttivo maggioritario ${ }^{10}$.

Il premio di maggioranza assegnato alla coalizione vincente attinge da una lista regionale bloccata (il cd. listino), di cui fanno parte il candidato alla carica di Presidente della Regione (che ne è capolista) e altri sei candidati (anziché 8 come in precedenza): metà uomini e metà donne inseriti in modo alternato. Chi è inserito nel listino regionale deve essere in corsa anche in un collegio provinciale; in caso di duplice elezione prevale quella avvenuta in ambito regionale, lasciando così spazio agli altri candidati presenti a livello provinciale. Si usufruisce del premio di maggioranza solo se la coalizione collegata al candidato presidente eletto non raggiunge almeno 42 seggi (il $60 \%$ dei seggi parlamentari) e solo nella misura in cui esso serva a raggiungere tale soglia ${ }^{11}$. I seggi eventualmente rimanenti vengono attribuiti all'opposizione, con il ripescaggio dei primi non eletti nei collegi provinciali. Il premio di maggioranza è, pertanto, eventuale, variabile e non (sempre) decisivo: eventuale, perché la prima distribuzione dei seggi può rivelarsi sufficiente ad assegnare una congrua maggioranza alla coalizione collegata al presidente eletto; variabile, perché il numero di seggi aggiuntivi, necessari a raggiungere i 42 seggi è legato al numero di seggi che la coalizione vincente ottiene mediante la ripartizione proporzionale; non decisivo, dato che quando scatta non sempre consente il raggiungimento della maggioranza assoluta dei seggi, non è, cioè, majority assuring: nella precedente legislatura, ad es., Rosario Crocetta divenne presidente con 39 seggi (su 90); pur aggiungendo ai 30 seggi proporzionali i 9 della lista regionale, ciò non consentì alla coalizione vincente di disporre di una maggioranza in aula.

Quanto alla soglia di sbarramento, cioè la soglia minima di voti richiesti a ciascuna lista per accedere in Assemblea, essa viene fissata al 5\%, una vera e propria rivoluzione se si tiene conto delle previsioni contenute nella disciplina transitoria del 2001, che prevedeva una barriera elastica, e tutto sommato penetrabile. Si tratta della più alta soglia esplicita in vigore nel nostro paese. Essa ha garantito una decisa riduzione della frammentazione: se nel 2001, le liste concorrenti erano state 20 e ben 18 avevano ottenuto almeno un seggio in Assemblea, nel 2006 le liste concorrenti saranno soltanto 12 e appena 8 entreranno in Assemblea.

Infine, quanto alla rappresentanza di genere, la legge prevede al fine di perseguire un maggiore equilibrio della rappresentanza fra i sessi, che tutti i candidati di ogni lista regionale dopo il capolista devono essere inseriti secondo un criterio di alternanza tra uomini e donne, e che nelle liste concorrenti a livello circoscrizionale nessuno dei due sessi possa essere presente in misura superiore ai due terzi del numero di candidati da eleggere nel collegio.

\section{L'offerta politica e la struttura della competizione}

La riduzione del numero dei deputati non ha scoraggiato la presentazione delle candidature, né fra i nuovi candidati né fra i parlamentari uscenti che hanno deciso di ricandidarsi ${ }^{12}$. In

gioni a statuto ordinario (legge n. 43/1995; legge cost. n. 1/1999), vale a dire quella particolare disciplina per cui in caso di sfiducia dell'esecutivo regionale si ritorna al voto.

${ }^{10}$ In particolare, la forma di governo neoparlamentare si basa su tre elementi essenziali: elezione diretta del presidente della regione, sistemi elettorali misti con premio di maggioranza, rapporto fiduciario tra esecutivo e legislativo con clausola simul stabunt, simul cadent.

${ }^{11}$ È stata impugnata dal Commissario dello Stato la norma che prevedeva la possibilità di eleggere in ogni caso tutti i candidati inseriti nel listino regionale.

${ }^{12}$ Quanto ai candidati deputati, ben 74 deputati uscenti si ricandidano: Cimino (Pdr Sicilia Futura) e Formica (Fi) ci riprovano per la sesta volta (entrambi sono all'Ars dal 1996), Ardizzone (Ap Centristi per Micari), Cracolici (Pd), Di Mauro (Idea Sicilia Popolari e Autonomisti), Laccoto (Pd), Savona (Fi) e Turano (Udc) inseguono il quinto mandato. Cfr. Regionali, in novecento in corsa per un seggio all'Ars, C. Reale, Repubblica.it Palermo, 7 ottobre 2017; I 20 posti in meno all'Ars non scoraggiano: 900 candidati su 15 liste, D. Gennaro, Corriere di Ragusa. 
prima battuta, novecento candidati concorrevano per 70 seggi, 15 liste supportavano 8 candidati alla presidenza.

In particolare, quanto ai candidati presidente, si passa ben presto da 8 a 5 candidati, dopo che i listini regionali di Franco Busalacchi di Noi Siciliani, Piera Lo Iacono della Lista civica per il lavoro e Pierluigi Reale di CasaPound Italia vengono ricusati dall'ufficio elettorale insediato presso la Corte d'Appello di Palermo. Le loro candidature vengono bocciate per irregolarità nella documentazione a corredo delle firme di sottoscrizione del listino. La bocciatura dei listini regionali dei tre candidati presidente fa cadere anche le liste presentate da Busalacchi, Lo Iacono e Reale nei vari collegi. In corsa per la successione di Rosario Crocetta restano: Giancarlo Cancelleri per il Movimento 5 Stelle, Claudio Fava per la lista Cento Passi per la Sicilia Fava Presidente, Roberto La Rosa per gli indipendentisti di Siciliani Liberi, Fabrizio Micari per il centrosinistra con il sostegno di 4 liste (Arcipelago Sicilia Movimento dei territori, Partito democratico, Pdr Sicilia Futura, Alternativa Popolare Centristi per Micari), Nello Musumeci per il centrodestra con il sostegno di ben 5 liste (DiventeràBellissima, Alleanza per la Sicilia Fratelli d'Italia-Noi con Salvini, Udc, Idea Sicilia Popolari e Autonomisti, Forza Italia). Le liste di Musumeci predominano numericamente, monopolizzando un terzo dell'offerta politica, un esercito di 310 candidati contro i 230 candidati del centrosinistra e i 62 candidati di Movimento 5 Stelle, Cento Passi per la Sicilia e Siciliani Liberi - si veda tab. 2.

TAB. 2 - Candidato presidente, liste regionali e liste provinciali collegate

\begin{tabular}{|c|c|c|}
\hline Candidato presidente & Liste regionali & Liste provinciali collegate \\
\hline Giancarlo Cancelleri & Movimento 5 Stelle (n. 1) & Movimento 5 Stelle \\
\hline Claudio Fava & $\begin{array}{l}\text { Cento Passi per la Sicilia Fava } \\
\text { Presidente (n. 2) }\end{array}$ & $\begin{array}{lll}\text { Cento Passi per la } & \text { Sicilia } \\
\text { (Mdp, Si, Possibile, Prc e } \\
\text { Verdi) }\end{array}$ \\
\hline Fabrizio Micari & Micari Presidente La Sfida Gentile (n. 3) & $\begin{array}{l}\text { Arcipelago Sicilia Movimento } \\
\text { dei territori, Partito democra- } \\
\text { tico, Pdr Sicilia Futura, Alter- } \\
\text { nativa Popolare Centristi per } \\
\text { Micari }\end{array}$ \\
\hline Nello Musumeci & $\begin{array}{l}\text { In Sicilia Nello Musumeci Presidente } \\
\text { (n. 4) }\end{array}$ & $\begin{array}{l}\text { DiventeràBellissima, Alleanza } \\
\text { per la Sicilia Fratelli d'Italia - } \\
\text { Noi con Salvini, Udc, Idea Si- } \\
\text { cilia Popolari e Autonomisti, } \\
\text { Forza Italia }\end{array}$ \\
\hline Roberto La Rosa & Siciliani Liberi (n. 5) & Siciliani Liberi \\
\hline
\end{tabular}

\subsection{Candidati alla presidenza: temi e strategie della campagna elettorale}

La prima candidatura ad essere presentata è quella di Giancarlo Cancelleri del Movimento 5 Stelle, già candidato presidente nelle elezioni regionali del 2012 e deputato all'Ars. La scelta del candidato è avvenuta sul web come da tradizione grazie al ricorso alle cosiddette regionarie che hanno consentito al M5s di presentare già il 9 luglio il proprio candidato alla presidenza e i propri candidati all'Ars ${ }^{13}$ : "Un'operazione di altissima democrazia e grandissimo coinvolgimento popolare" - la definiva l'allora deputato all'Ars Giancarlo Cancelleri "mentre gli altri partiti sono impegnati a discutere di nomi scelti a tavolino da poche persone all'interno delle segreterie dei partiti noi abbiamo avviato già da quattro mesi una discussione sul programma per una Sicilia migliore, che rilanci la nostra terra. Ed ora stiamo avviando un processo democratico che porterà centinaia di cittadini in giro per la Sicilia a dedicarsi anima e corpo per un progetto politico serio che culminerà il 9 luglio, quando al Castello a Mare annunceremo il nostro candidato alla presidenza della Regione" 14 .

${ }^{13}$ Il 19 giugno 2017 è iniziato ufficialmente il percorso che ha portato il M5s a presentare la sua lista e il suo candidato presidente alle elezioni per la Regione siciliana - lo annuncia un post del Blog delle Stelle: dal 19 al 25 giugno gli iscritti al M5s - entro il 1 luglio 2016 residenti in Sicilia con documento certificato - potevano proporre la propria candidatura per le elezioni regionali siciliane, compilando l'apposito form on line.

$14 \mathrm{Si}$ veda http://www.sicilia5stelle.it/2017/06/cancelleri-trionfo-della-democrazia-scelta-deicandidati-affidata-ai-cittadini-non-alle-segreterie-dei-partiti/, 19 giugno 2017. 
I nomi dei 62 attivisti del M5s che saranno inseriti nella lista presentata alle elezioni regionali del 5 novembre vengono resi noti sul blog di Beppe Grillo già il 5 luglio. Tra questi viene confermato (quasi) l'intero gruppo all'Ars: gli unici deputati regionali a non ripresentare la candidatura sono stati Giorgio Ciaccio e Claudia La Rocca, rinviati a giudizio per la vicenda delle firme false alle elezioni amministrative di Palermo del 2012. Con un secondo turno, è stato, poi, individuato, fra i 62 eletti il candidato presidente: ciascuno degli eletti doveva, infatti, entro $24 \mathrm{~h}$, esprimere la propria volontà di concorrere o meno anche per la candidatura alla presidenza della Regione ${ }^{15}$.

Le regionarie del M5s (primarie on line chiuse) possono essere analizzate prendendone in considerazione due aspetti: la partecipazione e la competitività (si veda tab. 3).

TAB. 3 - Partecipazione e competitività regionarie M5S 2017

\begin{tabular}{|l|c|c|c|c|c|c|}
\hline Regione & $\begin{array}{c}\text { Aventi } \\
\text { diritto }\end{array}$ & Votanti & $\begin{array}{c}\text { Tasso di partecipa- } \\
\text { zione }\end{array}$ & $\begin{array}{c}\text { Indice di attrattività della } \\
\text { competizione } \\
\text { Iturno }\end{array}$ & $\begin{array}{c}\text { Indice di attrattività della } \\
\text { competizione } \\
\text { II turno }\end{array}$ & $\begin{array}{c}\text { Indice di } \\
\text { competitività } \\
\text { II turno }\end{array}$ \\
\hline Sicilia & 6.787 & 4.350 & 64,1 & 0,90 & 0,89 \\
\hline
\end{tabular}

${ }^{1}$ Abbiamo calcolato il tasso di partecipazione partendo dall'unico dato ufficiale disponibile relativo al totale degli aventi diritto e proponendo una stima degli iscritti abilitati in Sicilia. Questa operazione è stata possibile ipotizzando che il numero di iscritti in ogni regione possa essere proporzionale al numero di elettori che hanno scelto il M5s durante l'ultimo appuntamento elettorale nazionale precedente le regionali (le elezioni europee del 2014). Nel nostro caso, sappiamo che alle ultime elezioni europee, sul territorio nazionale, hanno votato il M5s 5.792.865 elettori, di cui 448.539 in Sicilia e che gli iscritti nazionali al partito erano 87.656 ("iscritti certificati"); tramite una proporzione possiamo stimare in 6.787 il numero di iscritti nella regione. L'indice di attrattività della competizione è stato costruito come rapporto tra selezionati e selezionabili, esso varia tra un minimo di 0 (il numero dei selezionati è identico a quello dei selezionabili) e un massimo di 1 (dove il numero dei selezionati è pari a zero) - per ragioni di immediata leggibilità dell'indice si è deciso di sottrarre a 1 il rapporto, in questo modo più elevato è l'indice e più alto è il numero dei candidati alle primarie rispetto ai posti disponibili all'interno delle liste. L'indice di competitività di Kenig (2008), viene calcolato, infine, dividendo l'indice NEP di Laakso e Taagepera per il numero di candidati reali ed assume valori teorici compresi tra 0 e 1 - per il calcolo degli indici si veda Lanzone e Morini 2016.

Quanto alla prima, le regionarie sono state partecipate in media dal 64,1\% degli aventi diritto, gli "iscritti certificati" al M5s residenti in Sicilia entro il 1 luglio 2016 (o meglio una stima degli stessi, si veda la nota 1 in tab. 3), si tratta di uno dei tassi di partecipazione più alti comparati con l'ultima tornata di regionarie del ciclo di elezioni regionali 2014-15 (quando il tasso di partecipazione era variato nelle 9 regioni al voto, secondo Lanzone e Morini, tra il valore minimo del Veneto, 33\%, e quello massimo dell'Umbria, 66\%). L'indice di attrattività della competizione ci aiuta a valutare quanto sia stata intensa la corsa alla candidatura: al primo turno, l'indice è pari allo 0,90 , con un altissimo numero di candidati rispetto ai posti disponibili (626 candidati per 62 posti in lista), al secondo turno è pari allo 0,89 (dei 62 eletti 9 danno la loro disponibilità a concorrere per la candidatura alla presidenza della Regione). Infine, l'indice di competitività nella scelta del candidato alla presidenza indica un valore dello 0,35 a dimostrazione che la candidatura e la conseguente vittoria di Giancarlo Cancelleri non è stata caratterizzata da una serrata competizione. Alla votazione per il candidato presidente hanno partecipato 4.350 iscritti certificati e fra i 9 candidati in lizza, Giancarlo Cancelleri ha raccolto 2.224 voti, pari al 51,1\% dei consensi, seguito da Giampiero Trizzino al 17,1\% (742 voti), Francesco Cappello al 14,3\% (623 voti), Stefano Zito al 4,9\% (215 voti), Angela Foti al 4,8\% (211 voti) e gli altri (Tancredi, Listì Maman, Marano e Scarcella) tutti sotto il $3 \%{ }^{16}$.

${ }^{15}$ Cfr. Regionarie, ecco i 62 in lista. Confermato il gruppo all'Ars, S. Cataldo, LiveSicilia, 5 luglio 2017.

${ }^{16}$ Le regionarie verranno poi sospese a settembre 2017 dopo il ricorso presentato dall'attivista Mauro Giulivi che era stato escluso per non aver sottoscritto in tempo il Codice etico. La risposta del partito arriva attraverso un post sul blog di Beppe Grillo: "Il Movimento 5 Stelle in Sicilia ci sarà, non c'è nessun rischio caos. Il tribunale ha semplicemente accolto il ricorso di un iscritto che vuole essere in lista e, come misura cautelare, ha sospeso le regionarie. Ciò non toglie che il M5s parteciperà alle ele- 
Anche l'agenda della campagna elettorale è stata dominata dal M5s che ha imposto soprattutto due temi: quello dei candidati impresentabili e quello delle possibili irregolarità nelle operazioni elettorali (per approfondimenti si veda Sampugnaro e Montemagno 2017).

A meno di un mese dalle elezioni, il M5s pubblica una vera e propria lista dei candidati impresentabili, 19 impresentabili, 14 nelle liste del centrodestra che appoggiano Musumeci e 5 nelle liste di centrosinistra che appoggiano Micari. In un post apparso il 18 ottobre 2017, Giancarlo Cancelleri scrive: "Siamo molto preoccupati per la regolarità di queste elezioni. Nelle liste di Musumeci e Micari ci sono impresentabili, indagati e condannati e, da ultimo, anche un arrestato. Fra questi ci sono personaggi che si sarebbero macchiati proprio di reati legati al voto. Penso, ad esempio, alla condanna per corruzione elettorale o al processo per voto di scambio di due candidati che appoggiano Musumeci. Queste premesse possono pregiudicare il voto e i diritti dei siciliani". Dopo l'arresto del sindaco di Priolo, Antonello Riz$\mathrm{za}$, in corsa alle regionali per Forza Italia (14 ottobre), l'allora candidato premier e il candidato presidente del M5s, Luigi Di Maio e Giancarlo Cancelleri, arrivano a chiedere la presenza degli osservatori internazionali dell'Organizzazione per la sicurezza e la cooperazione in Europa (Osce) per il voto del 5 novembre perché vigilino sulle elezioni siciliane e fanno appello a Rosy Bindi, presidente della Commissione nazionale Antimafia perché velocizzi le operazioni di controllo sugli impresentabili in lista ${ }^{17}$.

Decisamente più complesse le vicende per la scelta del candidato presidente tanto della coalizione di centrodestra che della coalizione di centrosinistra.

Il centrodestra sceglie Nello Musumeci, presidente della Commissione regionale Antimafia, già candidato alla presidenza regionale nel 2006, a capo del suo movimento Alleanza Siciliana (ottenne il 5,3\% contro il 53,1\% del candidato di centrodestra Salvatore Cuffaro e il $41,6 \%$ della candidata del centrosinistra Rita Borsellino) e nel 2012, candidato della coalizione di centrodestra (ottenne il 25,7\%, sconfitto dal candidato di centrosinistra Rosario Crocetta, al $30,5 \%)^{18}$. La scelta di questa candidatura unitaria è stata, tuttavia, particolarmente travagliata.

Nel febbraio 2017 il suo movimento, DiventeràBellissima ${ }^{19}$, lo propone candidato alle primarie del centrodestra per la presidenza della Regione siciliana, primarie decise da tutte le forze politiche dello schieramento, ma poi annullate. Così, a fine aprile 2017, il movimento ufficializza la candidatura del suo leader, Nello Musumeci, alla presidenza. Il suo slogan è L'unico pizzo che piace ai siciliani, l'immagine che raffigura la sagoma e il pizzo di Musumeci evoca il programma del candidato che vuole puntare sulla legalità e sulla lotta alla corruzione. Tra luglio e agosto Fratelli d'Italia e Noi con Salvini, prima, e l'Udc, dopo, annunciano che presenteranno liste a sostegno di Musumeci. A sostegno del candidato arriveranno, poi, il cartello degli autonomisti (che mette assieme Cantiere Popolare di Saverio Romano, Mpa dell'ex presidente Raffaele Lombardo e Idea Sicilia dell'ex rettore dell'Università di

zioni, come previsto, e lo farà seguendo le decisioni che verranno prese dal tribunale. Il tour in Sicilia continua con il candidato presidente Giancarlo Cancelleri e Luigi Di Maio..." (cfr. Regionarie M5s, il Tribunale di Palermo sospende il voto su Cancelleri. Grillo: nessun rischio caos, Giornale di Sicilia, 12 settembre 2017; Regionali Sicilia: ecco chi è Giulivi, la spina nel fianco dei 5 Stelle, C. Reale, Repubblica.it Palermo, 19 settembre 2017). Alla fine Cancelleri otterrà il via libera dal Movimento per poter partecipare alla disputa elettorale: "Sono e rimango il candidato del M5s alla presidenza della Regione siciliana" (cfr. Regionali Sicilia, Cancelleri: "Sono e resto il candidato governatore", Repubblica.it Palermo, 13 settembre 2017).

${ }^{17}$ Cfr. Regionali Sicilia, Di Maio: "Vogliamo gli osservatori Osce per le elezioni”, C. Reale, Repubblica.it Palermo, 16 ottobre 2017; Regionali Sicilia, ispettori per vigilare sul voto? Minniti dice no, C. Reale, Repubblica.it Palermo, 18 ottobre 2017. La Commissione nazionale Antimafia si pronuncerà solo dopo le elezioni regionali, cfr. Impresentabili per l'Antimafia sono sei: da De Luca, unico eletto, a bracciante ibleo, M. Barresi, la Sicilia, 2 dicembre 2017.

${ }^{18} \mathrm{Nel} 2012$, il centrodestra vede candidato alla presidenza anche Gianfranco Miccichè, sostenuto dal Partito dei Siciliani - Mpa, Grande Sud, Nuovo Polo per la Sicilia Fli-Mps e Partito Pensiero Azione: il candidato Miccichè otterrà il 15,4\%.

${ }^{19}$ La costruzione della candidatura è addirittura antecedente ed è legata alla nascita del movimento DiventeràBellissima. Nel settembre 2014 Musumeci lascia, infatti, il movimento di Storace, La Destra, ed è tra $\mathrm{i}$ fondatori del movimento civico siciliano \#DiventeràBellissima, così denominato per richiamare una frase di Paolo Borsellino rivolta alla Sicilia, e di cui è leader. 
Palermo, Roberto Lagalla ${ }^{20}$ e Forza Italia. Solo a fine agosto Fi scioglie, infatti, la riserva, inizialmente indecisa tra il sostegno a Musumeci insieme a Fratelli d'Italia e Lega o la corsa solitaria a sostegno di Gaetano Armao, leader dei Siciliani Indignati, e preferito da Berlusconi nella corsa alla presidenza della Regione. Il centrodestra si compatterà, alla fine, sul nome di Nello Musumeci, con la previsione di un ticket tra il leader del movimento DiventeràBellissima e Gaetano Armao, già assessore ai beni culturali prima e al bilancio poi nei governi Lombardo ed ora designato, nel caso di vittoria del centrodestra, vicepresidente in pectore e assessore all'economia.

Nel centrosinistra, la scelta, dopo la rinuncia dell'allora presidente del Senato, Pietro Grasso, appare particolarmente difficile per il $\mathrm{Pd}$, su cui pesa, in modo più rilevante, l'eredità del governo Crocetta. Grasso sembrava, infatti, l'unico nome capace di aggregare le diverse anime del centrosinistra. Dopo la "garbata rinuncia" del Presidente Grasso ${ }^{21}$, si prospetta anche l'ipotesi primarie chieste anche dal presidente uscente che puntava a un secondo mandato, solo a fine agosto Leoluca Orlando propone la figura di Fabrizio Micari, rettore dell'Università di Palermo, come candidato civico di un centrosinistra che include il $\mathrm{Pd}, \mathrm{Ap}$ e Centristi per la Sicilia e che, fino all'ultimo, cerca una mediazione, poi fallita, con i bersaniani del Movimento democratico e progressista - Mdp e Sinistra Italiana - Si (indisponibili, tuttavia, fin dall'inizio a sostenere lo stesso candidato di Alfano). Successivamente dichiareranno l'appoggio a Micari Sicilia Futura, il movimento dell'ex ministro ed ex parlamentare Salvatore Cardinale, ed anche il presidente uscente Rosario Crocetta (i candidati del Megafono confluiranno nella lista Arcipelago Sicilia Micari Presidente) $)^{22}$.

La candidatura del rettore dell'Università di Palermo, il cui slogan La sfida gentile riecheggia la force tranquille di Mitterand, sconta tuttavia almeno tre problemi: anzitutto, l'eredità dell'amministrazione uscente, sulla quale pesa il giudizio negativo di molti elettori siciliani (il 78\%, secondo un sondaggio Demos \& Pi dell'ottobre 2017, si veda Atlante politico 69. Elezioni Regione Sicilia); in secondo luogo, la limitata notorietà del candidato alla presidenza che oltre un terzo degli intervistati ammette di non conoscere; infine, la presenza di una candidatura forte alla sua sinistra (Biorcio e Bordignon 2017).

A sinistra, la lista unitaria Cento Passi per la Sicilia candida, infatti, Claudio Fava, vicepresidente della Commissione nazionale Antimafia - tra i nomi circolati c'erano pure quelli del giornalista Corradino Mineo e dell'editore Ottavio Navarra per Rifondazione Comunista - al centro della campagna di Fava chiaramente il tema dell'antimafia per un candidato, come recita uno dei suoi slogan, Scandalosamente onesto. Secondo il candidato del centrosinistra, Fabrizio Micari, sono stati i vertici romani di Mdp e Si a non volere l'intesa col Pd facendo

${ }^{20}$ A fine settembre, anche Vittorio Sgarbi, che aveva annunciato la sua candidatura a presidente, sostenuto da Rinascimento e Moderati in Rivoluzione, si ritira e dichiara il proprio sostegno a Musumeci.

${ }^{21}$ Cfr. Regionali, Grasso chiude il dibattito: "Non mi candido, ho doveri stringenti". Confronto nel Pd, ipotesi primarie, Giornale di Sicilia, 20 luglio 2017; Pietro Grasso dice no: "Resto presidente del Senato, non mi candido alla Regione, La Sicilia, 20 luglio 2017.

${ }^{22}$ Rosario Crocetta, capolista, e gli altri 6 candidati nella lista Arcipelago-Micari Presidente a Messina verranno, poi, esclusi dalla competizione. I giudici hanno rilevato contraddizioni nei tempi di presentazione della lista da parte del delegato: "entro il termine previsto non risultava depositata né la lista dei candidati né la prescritta documentazione" recita il verbale notificato. Un problema non da poco per l'unica lista caratterizzata come lista Micari. La presenza a Messina, infatti, oltre ad essere simbolica per la candidatura del presidente uscente (che non è in corsa in nessun altro collegio), ha anche una valenza strategica: la provincia di Messina è la terza più popolosa, e Arcipelago Sicilia - che aveva già dovuto rinunciare alla lista siracusana - rischia senza i voti messinesi di non raggiungere la soglia di sbarramento, non approdando così all'Ars - cfr. Crocetta vede Renzi: verso il ritiro della candidatura in Sicilia, ilGiornale.it, 4 settembre 2017; Verso accordo tra Micari e Crocetta: si lavora alla "lista del presidente", CorrieredelMezzogiorno.it, 5 ottobre 2017; Regionali, Crocetta escluso dalle candidature. Micari: "Presenteremo subito ricorso", C. Reale, Repubblica.it Palermo, 8 ottobre 2017; Crocetta non potrà correre per le elezioni regionali in Sicilia. Il Tar respinge il ricorso: il governatore uscente non sarà candidato, La Stampa, 13 ottobre 2017. I Tar di Palermo e Catania respingeranno, poi, i due ricorsi presentati dal Comitato Micari e dall'ex governatore della Sicilia contro l'esclusione della lista Micari-Arcipelago a Messina, stabilita dall'ufficio elettorale circoscrizionale. 
così naufragare il campo largo, i dirigenti locali di quei partiti erano stati infatti fra i primi a convergere sul nome di Micari.

Alla competizione per la Presidenza della Regione siciliana partecipa anche Roberto La Rosa, del movimento indipendentista Siciliani Liberi, che punta ad essere la voce dei siciliani "stanchi di sentirsi colonizzati e defraudati" ${ }^{23}$, ma la sua candidatura alla presidenza appare come testimonianza di bandiera, di un movimento che difficilmente riuscirà a superare lo sbarramento.

\subsection{Liste e listini: adeguamento strategico e trasformismo}

Una delle principali novità delle elezioni del 2017 è stato il miglior adeguamento strategico delle liste alle regole elettorali. Rispetto alle regionali del 2012, quando la scheda elettorale presentava i nomi di 10 candidati alla presidenza e i simboli di 20 liste, si assiste infatti ad una decisa riduzione della frammentazione, frutto principalmente dell'accordo all'interno del centrodestra sul nome di Nello Musumeci, come candidato unitario alla presidenza e degli accorpamenti per cercare di superare la soglia di sbarramento del 5\%: così, a sinistra, per scongiurare l'errore commesso nel 2012 (2 liste oscillanti intorno al 3\%) si presenta una lista unitaria, Cento Passi per la Sicilia - Fava Presidente, che mette assieme Mdp, Si, Possibile, Prc e Verdi; nel centrosinistra, i centristi di Angelino Alfano corrono assieme agli uomini di Casini nella lista Alternativa Popolare Centristi per Micari e il Movimento politico di Crocetta confluisce nella lista Arcipelago Sicilia Movimento dei territori; nelle fila del centrodestra si aggregano, da un lato, le liste Fratelli d'Italia e Noi con Salvini nel cartello elettorale Alleanza per la Sicilia, e dall'altro, Lagalla, Romano e Lombardo trovano un accordo in una lista unica di centro, Idea Sicilia Popolari e Autonomisti.

Il miglior adeguamento strategico agli incentivi offerti dalla legge elettorale dà i suoi risultati (Emanuele e Riggio 2017). Anzitutto diminuisce in misura significativa il numero di liste provinciali, in una cornice, peraltro, non più bipolare ma tripolare (Bolgherini e Grimaldi $2015)^{24}$ : se nel 2012 le liste in gioco erano 20 ora diventano 12. Ma la novità più rilevante riguarda le liste che ottengono rappresentanza: se nel 2012 erano state solo 9 sulle 20 presentate (tra gli altri, rimasero fuori dall'Assemblea, la sinistra radicale, Italia dei Valori, Futuro e Libertà per l'Italia), nel 2017 delle 12 liste in gioco quasi tutte ottengono rappresentanza in Assemblea, le uniche liste a non conquistare almeno un seggio sono state le liste Alternativa Popolare Centristi per Micari, Arcipelago Sicilia Movimento dei Territori e Siciliani Liberi. Una disproporzionalità ridotta, quindi, con il 93\% dei voti validi rappresentati all'Ars; una disproporzionalità più bassa della affollata consultazione del 2012 e, soprattutto, delle elezioni anticipate del 2008 quando la disproporzionalità - calcolabile tramite l'indice di Gallagher - era balzata con poco meno di 12 punti percentuali nella "zona maggioritaria" con solo 4 liste delle 12 in gioco a superare la soglia e conquistare seggi all'Ars, grazie alla riproposizione a livello locale del formato del gioco nazionale, imperniato su due grandi partiti (Pdl e Pd) e con un ruolo pivotale - almeno in Sicilia - dei postdemocristiani di Mpa e Udc (le 4 liste assieme rappresentavano all'Ars il 78,6\% dei voti validi).

I dati smentiscono, inoltre, l'ipotesi di una "transumanza" dal centrosinistra al centrodestra che si presentava, almeno secondo i sondaggi, come il favorito, ovvero di un disastroso effetto bandwagon ai danni del governo uscente ${ }^{25}$. Se è vero che la XVI legislatura è stato un inno al trasformismo (complessivamente, i cambi sono avvenuti per 58 deputati, alcuni dei

${ }^{23}$ Cfr. Parte la campagna di Siciliani Liberi La Rosa candidato governatore, LiveSicilia, 28 agosto 2017.

${ }^{24}$ Le elezioni regionali siciliane del 2017 - come le elezioni del ciclo 2014-15 per le regioni a statuto ordinario - confermano la spaccatura della dinamica bipolare ed il passaggio ad una dinamica tripolare (in Sicilia il sistema si era mosso verso il tripolarismo già nelle elezioni regionali del 2012, con il successo del M5s, primo partito nell'isola).

${ }^{25}$ Per effetto bandwagon si intende la salita "sul carro del vincitore", ossia la tendenza di élite ed elettori a sostenere il candidato/partito ritenuto vincente, per approfondire si veda Noelle-Neumann (2002). 
quali hanno cambiato più volte nell'arco della stessa legislatura $)^{26}$, non vi è nessun movimento univoco verso il centrodestra a differenza di quanto alcuni analisti segnalano. In particolare, dei 90 deputati Ars, 16 non si sono ricandidati (si tratta di Cirone, Milazzo, Panarello, Raia e Rinaldi del Pd; Cascio, D'Asero e Fazio del Pdl; Fiorenza, Greco e Lombardo del Mpa; Nicotra e Dina dell'Udc; Ciaccio e La Rocca del M5s; Malafarina del movimento Il Megafono) e 30 ricandidati sono rimasti fedeli alla lista di elezione del 2012.

Degli altri 44 deputati: 21 si ripresentano in un partito diverso ma facente parte della stessa coalizione; gli altri 23 si ripresentano in un partito che sostiene una diversa coalizione ma, fra questi, 11 passano dal centrodestra al centrosinistra già durante la legislatura e si ricandidano in liste di centrosinistra (si tratta di Salvatore Cascio, Cimino, D'Agostino, Lo Giudice, Picciolo e Tamajo, passati al gruppo parlamentare Sicilia Futura col quale si ricandidano; Lantieri, Ruggirello e Sudano passati al gruppo Pd col quale poi si ricandidano; Fontana e Vinciullo passati ad Ap con cui poi si ricandidano); 9 passano nei mesi immediatamente precedenti le elezioni dal centrosinistra al centrodestra; 2 dal centrosinistra alla sinistra; 1 dal M5s al centrosinistra - si veda tab. 4 .

TAB. 4 - Ricandidature e trasformismi tra il 2012 e il 2017

\begin{tabular}{|c|c|c|c|}
\hline & Deputato & $\begin{array}{l}\text { Lista di elezione e candidato pre- } \\
\text { sidente sostenuto } 2012\end{array}$ & $\begin{array}{c}\text { Candidato nella lista e candidato } \\
\text { presidente sostenuto } 2017\end{array}$ \\
\hline \multirow[t]{11}{*}{ Dal CD al CS } & Cascio Salvatore & PID-Cantiere Pop. (Musumeci) & PDR-Sicilia Futura (Micari) \\
\hline & Cimino Michele & Grande Sud (Miccichè) & PDR-Sicilia Futura (Micari) \\
\hline & D'Agostino Nicola & Partito dei Sic. - Mpa (Miccichè) & PDR-Sicilia Futura (Micari) \\
\hline & Lo Giudice Salvatore & Musumeci Pres. (Musumeci) & PDR-Sicilia Futura (Micari) \\
\hline & Picciolo Giuseppe & Partito dei Sic. - Mpa (Miccichè) & PDR-Sicilia Futura (Micari) \\
\hline & Tamajo Edmondo & Grande Sud (Miccichè) & PDR-Sicilia Futura (Micari) \\
\hline & Ruggirello Paolo & Musumeci Pres. (Musumeci) & PD (Micari) \\
\hline & Sudano Valeria & PID-Cantiere Pop. (Musumeci) & PD (Micari) \\
\hline & Lantieri Annunziata & Grande Sud (Miccichè) & PD (Micari) \\
\hline & Fontana Vincenzo & PDL (Musumeci) & AP-Centristi per M. (Micari) \\
\hline & Vinciullo Vincenzo & PDL (Musumeci) & AP - Centristi per M. (Micari) \\
\hline \multirow[t]{9}{*}{ Dal CS AL CD } & Canì Gaetano & UDC (Crocetta) & UDC (Musumeci) \\
\hline & Giuffrida Salvatore & UDC (Crocetta) & Idea Sic. Pop. e Aut. (Musumeci) \\
\hline & La Rocca R. Margherita & UDC (Crocetta) & UDC (Musumeci) \\
\hline & Lentini Salvatore & UDC (Crocetta) & FI (Musumeci) \\
\hline & Ragusa Orazio & UDC (Crocetta) & FI (Musumeci) \\
\hline & Sorbello Giuseppe & UDC (Crocetta) & UDC (Musumeci) \\
\hline & Turano Girolamo & UDC (Crocetta) & UDC (Musumeci) \\
\hline & Coltraro Gianbattista & Il Megafono (Crocetta) & UDC (Musumeci) \\
\hline & Vullo Gianfranco & Il Megafono (Crocetta) & Idea Sic. Pop. e Aut. (Musumeci) \\
\hline \multirow[t]{2}{*}{ Dal CS alla $\mathrm{S}$} & Apprendi Giuseppe & PD (Crocetta) & 100Passi (Fava) \\
\hline & Maggio M. Leonarda & PD (Crocetta) & 100Passi (Fava) \\
\hline Dal M5s al CS & Venturino Antonio & M5s (Cancelleri) & Arcipelago Sicilia (Micari) \\
\hline
\end{tabular}

Fonte: dati Ars

Come si vede, nessuna transumanza che spinga i candidati dal centrosinistra al centrodestra; i cambi non hanno avuto, in realtà, confini: si sono attuati da destra a sinistra, gli unici (o quasi) che sono rimasti immuni - se si esclude il caso di Venturino - sono i componenti del M5s $\mathrm{s}^{27}$.

${ }^{26}$ Secondo i dati OpenArs, in merito ai cambi effettuati dai deputati anche nell'ambito delle modifiche delle denominazioni di tutti i gruppi parlamentari nel corso della XVI legislatura, ci sono stati: 75 cambi da maggioranza a maggioranza; 11 cambi da opposizione a opposizione; 17 cambi da opposizione a maggioranza; 4 da maggioranza a misto; 4 ripensamenti, 1 da maggioranza a opposizione ed 1 da opposizione a misto (cfr. Report \#OpenArs Edizione finale 2017; Report \#Cambiogruppo, report statistico relativo alle variazioni dei gruppi parlamentari Ars 2016).

${ }^{27} \mathrm{Ci}$ sono stati, poi, nell'ultima seduta, anche, dei cambi tecnici e la costituzione di nuovi gruppi all'Ars per evitare la raccolta di firme di alcune liste - 29 deputati su 90 hanno cambiato gruppo (cfr. Ars, cambiacasacca da record: 29 in un solo giorno, C. Reale, Repubblica.it Palermo, 20 settembre 2017; Ars, come si "cambia" per non morire, R. Pessina, Quotidiano di Sicilia, 29 settembre 2017), la legge regionale 29 del 1951 all'art. 13 prevede infatti che: "Nessuna sottoscrizione è richiesta per i 
Gli unici casi in cui si può parlare di un effetto bandwagon sono i 9 casi di deputati che dal centrosinistra passano al centrodestra, a poche settimane dal voto: Canì, Giuffrida, La Rocca, Lentini, Ragusa, Sorbello, Turano, Coltraro e Vullo - tutti, tranne Coltraro e Vullo ${ }^{28}$, eletti nella lista Udc nel 2012, lista che torna ora nella sua collocazione "naturale", appoggiando il candidato alla presidenza della coalizione di centrodestra ${ }^{29}$ a fianco dell'altra lista di centro dei Popolari e Autonomisti, e rendendo come vedremo ancora più pesante la sconfitta del candidato di centrosinistra. Ad appoggiare Micari rimarrà, dopo la rottura con l'Udc, la componente dei Centristi di Casini (che si allea con Alternativa Popolare di Angelino Alfano) e Pdr Sicilia Futura.

In particolare, fra le quattro liste di centro: l'Udc di Cesa confluisce, dopo la rottura con Pier Ferdinando Casini, nella lista Musumeci Presidente-Sicilia Vera-Libertas-Rete DemocraticaUnione di Centro ${ }^{30}$; il cartello elettorale degli autonomisti, Idea Sicilia Popolari e Autonomisti, aggrega l'Mpa dell'ex presidente Raffaele Lombardo, Cantiere Popolare di Saverio Romano e Idea Sicilia di Roberto Lagalla, già indicato come futuro assessore dal candidato presidente del centrodestra; a conferma della natura "trasversale" di quella tradizione la lista Pdr-Sicilia Futura-Psi-Micari Presidente sostiene Micari, ma l'ex ministro ed ex parlamentare Salvatore Cardinale (69 anni, un tempo vicino a Calogero Mannino, una figlia - Daniela alla Camera a cui ha trasferito i consensi), a spoglio ancora aperto ha fatto capire che potrebbe schierare i suoi eletti per "rinforzare" la maggioranza di Musumeci in bilico con i suoi 35 deputati: "Tutto quello che andrà bene per la Sicilia noi lo voteremo"31.

L'unica lista di centro a non entrare all'Ars è la lista Alternativa Popolare Centristi per Micari, fermatasi al 4,2\%, ciò ha comportato alcune eccellenti non rielezioni: clamorosa la mancata rielezione di Giovanni Ardizzone, ex presidente dell'Ars, candidato come capolista a Messina nella componente dei Centristi per la Sicilia (ma anche degli uscenti Forzese, Vinciullo e Fontana). Entrambe le componenti pagano probabilmente l'ambiguità della loro posizione politica. Alternativa Popolare di Angelino Alfano è stata combattuta sulla posizione da tenere: rimanere nel centrosinistra o tornare al centrodestra. Il coordinatore di Fi, Gianfranco Miccichè, si è impegnato da parte sua fino all'ultimo nel tentativo di arrivare ad un'intesa con Alfano, ad un dialogo coi centristi; una trattativa complicata su cui hanno pesato, però, sin dall'inizio i veti irremovibili di FdI e Lega. I Centristi per l'Europa di Casini, in una prima fase, si dicevano pronti addirittura a lanciare un loro candidato alla presidenza della Regione, individuandolo nel senatore Giampiero D'Alia, coordinatore nazionale dei Centristi per l'Europa. I Centristi chiedevano, soprattutto, un segnale di discontinuità al Pd, lo stesso Pier Ferdinando Casini parlando a margine dell'assemblea regionale del partito (luglio 2017) sottolineava: "In questo momento non si dà per scontato nulla anche perché non sappiamo il centrosinistra cosa propone in Sicilia. Siamo in attesa di capire. È chiaro che noi chiediamo una discontinuità rispetto al governo Crocetta perché da questa giunta siamo usciti e sarebbe assurdo riproporla" ${ }^{, 2}$.

partiti o gruppi politici costituiti presso l'Assemblea regionale in gruppo parlamentare nella legislatura precedente o che nell'ultima elezione regionale abbiano ottenuto almeno un seggio".

28 Gianfranco Vullo e Giambattista Coltraro, eletti nel 2012, col Megafono di Crocetta, Movimento politico Crocetta Presidente passano, rispettivamente, al Pd e al gruppo Sicilia Democratica per le riforme già nel dicembre 2012. Vullo si candida poi nella lista Idea Sicilia Popolari e Autonomisti e Coltraro nella lista dell'Udc, entrambi si trovano a sostenere il candidato presidente sconfitto 5 anni fa, Nello Musumeci (cfr. Dal centrosinistra a Musumeci. Regionali saltafosso last minute, LiveSicilia, 11 novembre 2017).

${ }^{29}$ Non si può dare una tale chiave di lettura per i passaggi di Apprendi e Maggio dal Pd alla lista CentoPassi per la Sicilia per sostenere la candidatura di Claudio Fava, o per il passaggio di Venturino dal M5s alla lista Arcipelago Sicilia.

${ }^{30}$ Cfr. L'Udc sostiene Musumeci, Cesa: “Discontinuità con Crocetta”, Giornale di Sicilia, 6 settembre 2017.

${ }^{31}$ Cfr. È boom di voti per Sicilia Futura, la lista dell'ex ministro Cardinale ha gli stessi che mancano al candidato presidente?, BlogSicilia.it, 6 novembre 2017.

${ }^{32}$ Cfr. Regionali, $i$ Centristi puntano su Gianfranco D'Alia per la presidenza, Giornale di Sicilia, 18 luglio 2017; cfr. Casini: “Alleanze per le Regionali? Serve discontinuità con Crocetta”, LiveSicilia, 17 luglio 2017. 


\section{I risultati elettorali}

I risultati delle elezioni regionali del 5 novembre 2017 sembrano confermare un orientamento stabile dell'elettorato siciliano verso i partiti di centrodestra (Cerruto e Raniolo 2009), come dimostra l'avvenuta elezione del Presidente Nello Musumeci con la sua maggioranza parlamentare di riferimento. In questa direzione sono andati i primi commenti dei dati elettorali siciliani, molto più interessati a costituire una proiezione per la prossima competizione nazionale che a sottolineare gli elementi di specificità del contesto politico siciliano.

Si tratta di elementi di originalità che, però, non sembrano avere sostanzialmente modificato il comportamento elettorale dei siciliani (Dandoy e Schakel, 2013). I dati elettorali delineano sia sotto il profilo strutturale - legato alla natura e alle caratteristiche dei "blocchi", o poli coalizionali, che segnano la fine del bipolarismo - sia sotto il profilo della portata sistemica del voto siciliano - con riferimento ai temi della campagna elettorale - alcuni elementi di originalità a partire dagli spazi elettorali che oggi occupano i partiti e i movimenti che costituiscono veri e propri schieramenti alternativi.

\subsection{La partecipazione}

Il primo elemento di riflessione riguarda la partecipazione al voto dei siciliani. Le elezioni regionali siciliane - in quanto elezioni di secondo ordine - si caratterizzano per una bassa partecipazione rispetto al dato sulla partecipazione alle elezioni politiche ma sottolineano (ancora una volta) la bassa propensione dei siciliani alla partecipazione elettorale ${ }^{33}$ rispetto al resto del paese. Questa caratteristica è molto evidente nel raffronto dei dati sulla partecipazione relativi alle elezioni regionali a partire dal 2006, attraverso il quale è possibile rilevare una caratteristica tutta siciliana di una minore propensione alla partecipazione alle elezioni regionali rispetto ad altre competizioni elettorali.

Nel complesso i dati sulla partecipazione elettorale evidenziano, da un lato, l'incapacità della elezione diretta del presidente della Regione di incidere sulla bassa propensione dei siciliani a recarsi alle urne, dall'altro lato, l'andamento significativamente decrescente della percentuale media della partecipazione su base regionale - tranne che nelle elezioni regionali del $2008(66,7 \%)$ dove però ha molto influito la concomitanza con le consultazioni politiche come sottolineato, in modo particolare, dalle elezioni regionali del 2012 (47,4\%) e del 2017 $(46,7 \%)$.

In quest'ultimo caso, i dati riportati confermano anche una elevata varianza interna alla medesima macro-zona geopolitica se si tiene conto della differenza di 14 punti percentuali tra la provincia con la più alta percentuale di partecipazione (Messina) e la provincia con la percentuale più bassa (Enna).

TAB. 5 - Partecipazione elettorale nelle elezioni regionali in Sicilia (2006-2017); val. \%

\begin{tabular}{|l|c|c|c|c|c|}
\hline & Regionali 2001 & Regionali 2006 & Regionali 2008 & Regionali 2012 & Regionali 2017 \\
\hline Agrigento & 58 & 53 & 56,3 & 31,3 \\
\hline Caltanissetta & 55 & 49,5 & 56,5 & 41,4 \\
\hline Catania & 66,3 & 62,1 & 71 & 51,1 \\
\hline Enna & 54 & 49,8 & 54,5 & 41,7 \\
\hline Messina & 66,5 & 61,5 & 69,8 & 51,2 \\
\hline Palermo & 65,5 & 61,7 & 69,1 & 46 \\
\hline Ragusa & 65,4 & 62,9 & 70,3 & 49,3 \\
\hline Siracusa & 59,8 & 57,1 & 66,4 & 49,6 \\
\hline Trapani & 65,9 & 59,9 & 68,2 & 48,8 \\
\hline Sicilia & 63,5 & 59,2 & 66,7 & 47,5 \\
\hline
\end{tabular}

Le regionali del 2017 evidenziano delle differenze territoriali, a livello provinciale, nella partecipazione al voto. Le province di Catania $(51,6 \%)$ e Messina $(51,8 \%)$ sono ben al di sopra della media regionale $(46,7 \%)$. Le province di Ragusa (47,3\%), Siracusa $(47,6 \%)$, Palermo $(46,4 \%)$ e Trapani $(45,4 \%)$ sono sulla media regionale, mentre le province di Enna $(37,7 \%)$,

${ }^{33}$ L'astensionismo siciliano è stato considerato come una conferma dell'esistenza di un dualismo elettorale tra zone geopolitiche del paese: una vera e propria frattura territoriale che divide le zone del Centro-Nord da quelle del Sud Italia. 
Agrigento $(39,6 \%)$ e Caltanissetta $(39,8 \%)$ registrano dei valori percentuali ben al di sotto della media regionale.

Ed ancora, nonostante nel 2017 si registri un calo nella partecipazione rispetto alle elezioni regionali del 2012, è singolare l'aumento delle stesse percentuali nelle province di Catania, Messina e Palermo. Questo dato è in perfetta corrispondenza con le percentuali sulla partecipazione nelle città capoluogo di provincia che si attestano oltre la soglia del $50 \%$, tranne per il caso di Trapani (43,6\%). Sotto questo punto di vista, nel complesso la partecipazione elettorale a livello comunale mostra una corrispondenza diretta tra ampiezza del comune (rispetto al numero di abitanti) e percentuali della partecipazione al voto: più piccoli sono i comuni più basse sono le percentuali di partecipazione al voto.

A completamento del quadro sulla partecipazione elettorale nelle elezioni regionali del 2017 abbiamo osservato il cosiddetto astensionismo "attivo" ${ }^{34}$, vale a dire i voti non validi (schede bianche e schede nulle). Rispetto al totale dei voti inespressi questo dato evidenzia una diminuzione complessiva di più di 2 punti percentuali rispetto alla precedente competizione del 2012 e una diminuzione a quasi un terzo rispetto al dato fatto registrare nel 2008 e nel 2006. Si diversifica invece la composizione interna della componente voti inespressi: alla crescita compresa tra i 10 e i 15 punti percentuali del numero degli astenuti nelle ultime due elezioni regionali (astensionismo "passivo") corrisponde una sensibile diminuzione delle percentuali dei voti non validi (astensionismo "attivo"), rispetto al 4,5\% del 2012 nel 2017 si passa al $2,3 \%$ (si veda la tab. 6 ).

TAB. 6 - Astenuti, voti nulli e schede bianche nelle elezioni regionali (2001-2017), \% elettori

\begin{tabular}{|l|c|c|c|c|}
\hline & 2001 & 2006 & 2008 & 2012 \\
\hline Astenuti & 36,5 & 40,8 & 33,3 & 48,1 \\
\hline Schede nulle & 6,5 & & 5017 & 3,8 \\
\hline Schede bianche & 1 & 5,3 & 7,8 & 0,7 \\
\hline Totale voti inespressi & 44 & 46,1 & 41,1 & 0,3 \\
\hline * Il totale dei voti inespressi è uguale alla somma degli astenuti, delle schede nulle e delle schede bianche calcolati sul totale degli elettori.
\end{tabular}

Fonte: Archivio del Servizio Elettorale della Regione Siciliana

I dati sulla partecipazione rappresentano anche una cartina di tornasole rispetto allo sforzo messo in campo da tutti i partiti per ridimensionare la deriva dell'astensione dal voto che aveva, per certi versi, contaminato il risultato elettorale del 2012. In particolare, il M5s e il suo candidato alla presidenza della Regione avevano puntato sull'obiettivo di riportare i siciliani al voto. I dati complessivi sulla partecipazione, come abbiamo avuto modo di vedere, hanno evidenziato il fallimento nell'obiettivo di arginare il fenomeno dell'astensionismo. Non solo. Alcune analisi sui flussi elettorali ${ }^{35}$ sembrano addirittura evidenziare come sia il candidato di centrodestra Musumeci ad avere attinto di più, rispetto agli altri candidati, dal bacino dell'astensione.

\subsection{Il voto ai partiti}

I risultati elettorali delle elezioni regionali del 5 novembre 2017 confermano come la competizione elettorale si sia giocata su due diversi piani: quello delle circoscrizioni elettorali, e dunque le liste provinciali, che contribuisce alla ripartizione della maggior parte dei seggi all'Ars (62 su 70); e quello delle liste regionali bloccate a cui corrispondono i candidati alla carica di presidente della Regione (che ne sono i capolista) e che concorrono per l'assegnazione su base maggioritaria di altri 7 seggi all'Ars. Da questa ripartizione viene escluso il seggio riservato al candidato presidente secondo classificato.

\footnotetext{
${ }^{34}$ La distinzione, oggi in disuso, tra astensionismo "passivo", o in senso stretto, e "attivo" o "civico", comprensivo delle schede bianche e voti nulli, si deve alla scuola francese di studi elettorali, in particolare si veda Lancelot (1968). Per un quadro sul dibattito e le ricerche fino agli anni '70 si veda Caciagli e Scaramozzino (1983).

${ }^{35}$ Il riferimento è quello dell'elaborazione dei flussi fatta dall'Istituto Cattaneo.
} 
TAB. 7 - Voti validi (\%) ai partiti e numero dei seggi (v.a.) nelle elezioni regionali (2006-2017)

\begin{tabular}{|c|c|c|c|c|c|c|c|c|}
\hline & \multicolumn{2}{|c|}{2006} & \multicolumn{2}{|c|}{2008} & \multicolumn{2}{|c|}{2012} & \multicolumn{2}{|c|}{2017} \\
\hline & voti & seggi & voti & seggi & voti & seggi & voti & seggi \\
\hline Pdl & - & - & 33,5 & 34 & 12,9 & 12 & - & - \\
\hline $\mathrm{Pd}$ & - & - & 18,8 & 29 & 13,4 & 14 & 13 & 11 \\
\hline $\mathrm{Pds} / \mathrm{Ds}$ & 14 & 15 & - & - & - & - & - & - \\
\hline An & 10,6 & 10 & - & - & - & - & - & - \\
\hline $\mathrm{Fi}$ & 19,2 & 16 & - & - & - & - & 16,3 & 12 \\
\hline Msi-Ft/La Destra-Ft & 0,3 & - & 1,4 & - & - & - & - & - \\
\hline Idv & - & - & 1,8 & - & 3,5 & - & - & - \\
\hline Margherita Dl & 12 & 15 & - & - & - & - & - & - \\
\hline Udc & 13 & 13 & 12,5 & 11 & 10,8 & 11 & 7 & 5 \\
\hline Mpa-Ns & 12,5 & 10 & 13,9 & 16 & 9,5 & 9 & - & - \\
\hline Alleanza Siciliana & 2,4 & - & - & - & - & - & - & - \\
\hline L'Aquilone & 5,7 & 5 & - & - & - & - & - & - \\
\hline $\mathrm{Us} / \mathrm{Sa}$ & 5,2 & 5 & 4,9 & - & - & - & - & - \\
\hline Lombardo Pres. & - & - & 4,5 & - & - & - & - & - \\
\hline Finocchiaro Pres. & - & - & 3,1 & - & - & - & - & - \\
\hline M5s & - & - & - & - & 14,9 & 15 & 26,7 & 19 \\
\hline Claudio Fava Pres./Sel/Verdi & - & - & - & - & 3,1 & - & - & - \\
\hline Futuro e Libertà per l'Italia & - & - & - & - & 4,4 & - & - & - \\
\hline Grande Sud Miccichè & - & - & - & - & 6 & 5 & - & - \\
\hline Il Megafono & - & - & - & - & 6,2 & 5 & - & - \\
\hline Nello Musumeci Pres. & - & - & - & - & 5,6 & 4 & - & - \\
\hline Pid-Cantiere popolare & - & - & - & - & 5,9 & 5 & - & - \\
\hline Cento Passi per la Sicilia & - & - & - & - & - & - & 5,2 & 1 \\
\hline Arcopelago Sicilia-Micari Pres. & - & - & - & - & - & - & 2,2 & - \\
\hline Pdr-Sicilia Futura & - & - & - & - & - & - & 6 & 2 \\
\hline Alternativa Popolare & - & - & - & - & - & - & 4,2 & - \\
\hline $\begin{array}{l}\text { Alleanza per la Sicilia/Nello Musume- } \\
\text { ci Pres./Fratelli d'Italia/Noi con Salvini }\end{array}$ & - & - & - & - & - & - & 5,6 & 3 \\
\hline DiventeràBellissima & - & - & - & - & - & - & 6 & 4 \\
\hline Idea Sicilia/Popolari e Autonomisti & - & - & - & - & - & - & 7,1 & 5 \\
\hline Altri & 5 & 1 & 5,6 & - & 3,8 & - & 0,7 & - \\
\hline Totali & 100 & 90 & 100 & 90 & 100 & 80 & 100 & 62 \\
\hline
\end{tabular}

Quanto ai risultati ottenuti dalle liste provinciali, il primo dato rilevante che emerge è l'avanzamento del M5s (26,7\%) di quasi 12 punti percentuali rispetto alle regionali del 2012. Questo risultato ha confermato il M5s come primo partito all'Ars con un aumento considerevole dei seggi assegnati rispetto al 2012 (20 su 15), tenuto conto sempre della riduzione del numero complessivo dei parlamentari. La valenza della performance elettorale del M5s è ancora più evidente se si guarda alla differenza $(-10,4 \%)$ con la seconda lista più votata Forza Italia $(16,3 \%)$ e il Partito Democratico che si ferma al $13 \%$.

Il secondo dato politico rilevante è costituito dal mancato superamento dello sbarramento del 5\% da parte del partito dell'ex Ministro degli Esteri Angelino Alfano che da sempre aveva fatto della Sicilia la sua roccaforte elettorale. Alternativa popolare, nell'intenzione del suo leader, aveva l'obiettivo di occupare lo spazio al centro del sistema politico siciliano essendo esso stesso costituito per lo più da transfughi del Popolo della libertà e dell'Udc, nonostante il cambio di schieramento politico e l'essere diventato integrale alla coalizione di centrosinistra sia a livello nazionale che a livello regionale.

Infine, il terzo dato politico è rappresentato dal ritorno (dopo 16 anni) tra gli scranni dell'Ars della sinistra radicale rappresentata nella campagna elettorale dalla lista Cento Passi per la Sicilia $(5,2 \%)$ che è riuscita a superare lo sbarramento. Insieme a questi conquista un seggio per la prima volta in assoluto la Lega attraverso il cartello elettorale ${ }^{36}$ Alleanza per la Sicilia $(5,6 \%)$ che conquista complessivamente 3 seggi.

Sono queste le principali considerazioni sui risultati elettorali, tra l'altro, largamente condivise dagli analisti. Ma se si va più in profondità, guardando a quanto accaduto nelle diverse circoscrizioni elettorali provinciali, il dato elettorale è meno omogeneo nella sua composizione di quanto appare.

${ }^{36}$ Per "cartello elettorale" si intende quell'insieme di liste di cui almeno una nazionale, ecco perché possono essere ricomprese nella categoria liste nazionali, oltre ai partiti nazionali. Essi vengono, dunque, distinti dalle liste regionali (o locali) e liste personali, ossia quelle presenti in un'unica realtà regionale (Bolgherini e Grimaldi, 2015). 
A tale proposito, è necessario fare una premessa circa alcune delle caratteristiche storiche della rappresentanza in Sicilia, secondo le quali sono i candidati eletti a costituire il vero trait d'union tra elettori e partiti, specie a livello locale. Sostanzialmente, in Sicilia ha prevalso la rappresentanza individualistica vs. la rappresentanza partitica, con tutto quello che ne consegue: frammentazione dell'offerta politica; dispersione delle preferenze tra più candidati ed eletti; formazione di "partiti personali" e relativa debolezza nei processi di istituzionalizzazione dei partiti (Cerruto, 2008). In Sicilia sembra prevalere, ancora una volta, la configurazione di un sistema politico fatto da "partiti nel territorio" ${ }^{37}$; vale a dire, un sistema di rappresentanza candidate-oriented basato su un reticolo di rapporti personali e su appartenenze culturali che danno vita a correnti e gruppi interni all'organizzazione del partito.

A sostegno di questa tesi i dati relativi alle principali liste prendendo a riferimento le diverse circoscrizioni provinciali. Forza Italia - nonostante apparentemente ${ }^{38}$ abbia ottenuto un risultato elettorale migliore rispetto al Popolo della Libertà delle precedenti elezioni regionali - è in provincia di Messina che ha avuto un differenziale più alto $(+11,6 \%)$ rispetto al 2012 . Il differenziale più basso riscontrato, invece, è quello della provincia di Agrigento $(-3,5 \%)$. Analizzando politicamente questi dati non è lontano dalla realtà sostenere che il risultato di Fi in provincia di Messina sia legato alle vicende locali come il passaggio di Francantonio Genovese - in seguito alle sue vicende giudiziarie - dal Partito Democratico a Forza Italia e la conseguente candidatura del figlio, appena maggiorenne, Luigi Genovese nella lista provinciale di Fi. Così come, il risultato negativo di Fi in provincia di Agrigento è legato alla positiva performance elettorale di Alternativa Popolare $(8,6 \%)$ dell'agrigentino Angelino Alfano schierato a sostegno del candidato di centrosinistra Micari e che nel 2012 invece militava nel Pdl.

Anche il Pd che sembra avere mantenuto, in linea di massima, le stesse percentuali di voti del 2012, evidenzia sostanziali disomogeneità nei risultati ottenuti su base provinciale. Il differenziale più alto rispetto ai risultati del 2012 è quello rilevato in provincia di Enna $(+6,6 \%)$, mentre il differenziale più basso è quello rilevato in provincia di Messina $(-7,5 \%)$. In quest'ultimo caso ha influito certamente la vicenda Genovese che ha fortemente condizionato il risultato del Pd messinese, mentre il risultato positivo del Pd in provincia di Enna è legato, tra l'altro, alla riabilitazione politica dell'ex sen. Crisafulli a cui da decenni sono legate le principali vicende politiche dell'ennese.

Più omogeneo su base provinciale è il calo dell'Udc. Si tratta di un calo generalizzato intorno ai 4 punti percentuali - con delle punte massime rilevate in provincia di Catania $(-7,7 \%)$ ed in provincia di Ragusa $(-5,5 \%)$ - frutto, da un lato, della scelta politicamente "forte" di riaccreditarsi dentro la coalizione di centrodestra, dopo l'esperienza a sostegno del governatore Crocetta, dall'altro lato, del proliferare di liste a valenza per lo più locale che politicamente si collocano nell'area di centro, spazio politico da tempo occupato dall'Udc quale erede naturale della Democrazia Cristiana.

L'analisi dei dati provinciali ci dice anche dell'altro che possiamo classificare sotto l'etichetta della caratterizzazione fortemente localistica di alcune liste e movimenti, fondamentalmente, a sostegno dei due candidati di centrodestra e centrosinistra. A sostegno del candidato di centrosinistra Fabrizio Micari si è schierata la lista Arcipelago Sicilia che ha conseguito un buon risultato soprattutto nel palermitano $(6,6 \%)$, anche se non ha superato lo sbarramento e la lista Pdr-Sicilia futura che fa riferimento all'ex ministro Cardinale che ottiene tanti consensi soprattutto nell'agrigentino (7,9\%), nel nisseno $(7,7 \%)$, nel palermitano $(7,4 \%)$ e nel messinese $(6,7 \%)$ e che riesce a conquistare 2 seggi all'Ars. A sostegno del candidato di centrodestra Musumeci invece ritroviamo: la lista DiventeràBellissima che ottiene un ottimo risultato soprattutto in provincia di Ragusa $(13,6 \%)$ e in provincia di Caltanissetta (8,6\%); la lista Idea Sicilia che raggruppa alcuni moderati fuoriusciti dall'Udc e i su-

${ }^{37}$ Secondo Diamanti (2003) la politica dei partiti nel nostro Paese, e conseguentemente in Sicilia, può essere letta attraverso tre diverse configurazioni: a) i partiti nel territorio (i post-democristiani); b) i partiti senza territorio (FI); c) il territorio contro la politica (MPA e AS).

${ }^{38}$ Il centro-destra nel 2012 si è presentato diviso con due candidati Nello Musumeci e Gianfranco Miccichè ai quali si sono collegate alcune liste (Grande Sud Miccichè, Futuro e Libertà per l'Italia) che hanno drenato molti voti (complessivamente più del 10\%) dal partito di riferimento, il Popolo della Libertà. 
perstiti del Movimento per l'Autonomia di Raffaele Lombardo che ottiene un buon risultato elettorale soprattutto in provincia di Agrigento $(12,9 \%)$ e in provincia di Palermo, città dell'ex rettore dell'Università di Palermo Lagalla che ne è stato l'ispiratore.

Delle vicende locali ha risentito anche il M5s che nonostante l'ottima performance sul piano regionale ha registrato una varianza molto alta in punti percentuali a livello provinciale. Una variazione percentuale inversamente proporzionale al grado di competitività rappresentato dai "signori delle preferenze", anche in riferimento al minore ricorso allo strumento della preferenza. Il differenziale più alto rispetto alle elezioni regionali del 2012 il M5s lo ottiene in provincia di Siracusa $(+19,3 \%)$ e, a seguire, in provincia di Enna $(+18,3 \%)$ e in provincia di Agrigento $(+15,7 \%)$; mentre il differenziale più basso lo registra in provincia di Palermo $(+8,6)$, in provincia di Ragusa $(+9,3 \%)$ e in provincia di Caltanissetta $(+9,7)$.

La personalizzazione della politica in Sicilia è un tratto caratteristico che si evidenzia anche nella "presidenzializzazione" del sistema di governo regionale. L'introduzione dell'elezione diretta del Presidente della Regione, a partire dal 2001, oltre a modificare il sistema di investitura, ha trasformato sostanzialmente la forma di governo ${ }^{39}$ concentrando nelle mani dell'organo monocratico tanto potere e altrettante prerogative in grado di condizionare anche il dibattito politico orientandolo sulla scelta dei candidati alla presidenza e sul nodo strategico delle alleanze elettorali.

Ecco perché risulta fondamentale completare il quadro sulle elezioni regionali del 2017 analizzando i dati sulle liste regionali, vale a dire sui capilista candidati alla presidenza della Regione. Le riflessioni che ne scaturiranno costituiscono, come avremo modo di vedere, una fondamentale chiave di lettura per la interpretazione di tutto il processo elettorale.

Le elezioni regionali del 2017 hanno visto competere 5 candidati per la presidenza della Regione, numericamente la metà rispetto alla precedente competizione elettorale del 2012. Ad avere la meglio è stato il candidato della coalizione di centrodestra Musumeci (con il 39,8\% dei consensi) che ha avuto la meglio sul candidato del M5s Cancelleri (che ha ottenuto il $34,6 \%$ dei consensi). Micari, candidato del centrosinistra, erede della coalizione che nelle regionali del 2012 aveva visto l'affermazione del governatore uscente Crocetta è giunto terzo con il $18,6 \%$. Anche se i dati riportati nella tabella sembrerebbero dimostrare che si sia trattato di un vero e proprio ballottaggio tra i primi due classificati, con il candidato di centrosinistra mai in corsa, una diversa aggregazione di essi evidenzia, ancora una volta, una "tripolarizzazione" del consenso con un rovesciamento delle gerarchie stabilite nelle precedenti elezioni del 2012 e un rafforzamento dei partiti di destra e di sinistra che tornano ad avere rappresentanza all'interno dell'Ars (Bolgherini e Grimaldi, 2015).

${ }^{39}$ Solitamente tra le due dimensioni opera una "legge" di congruenza. Ad una investitura forte, o diretta, corrispondono poteri altrettanto forti, o competenze (su questi temi si vedano Caciagli e Di Virgilio, 2005). 
TAB. 8 - Differenza tra $i$ voti validi solo per $i$ candidati presidente e le coalizioni rispetto alle regionali del 2012

\begin{tabular}{|c|c|c|c|c|c|c|}
\hline \multicolumn{3}{|c|}{ Elezioni regionali 2012} & \multicolumn{3}{|c|}{ Elezioni regionali 2017} & \multirow{3}{*}{$\begin{array}{c}\text { Diff. } \\
2017 / 2012 \\
16,4 \\
\end{array}$} \\
\hline \multicolumn{2}{|c|}{ Candidati e liste della coalizione } & \multirow{2}{*}{\begin{tabular}{c|}
$\%$ \\
voti \\
18,2
\end{tabular}} & \multicolumn{2}{|c|}{ Candidati e liste della coalizione } & \multirow{2}{*}{\begin{tabular}{|c|}
$\%$ \\
voti \\
34,6 \\
\end{tabular}} & \\
\hline Cancelleri & & & Cancelleri & & & \\
\hline & M5s & 14,9 & & M5s & 26,7 & 11,8 \\
\hline \multirow[t]{6}{*}{ Crocetta } & & 30,5 & Micari & & 18,6 & $-11,9$ \\
\hline & $\mathrm{Pd}$ & 13,4 & & $\mathrm{Pd}$ & 13 & \\
\hline & Udc & 10,8 & & Arcopelago Sicilia-Micari Pres. & 2,2 & \\
\hline & Il Megafono & 6,2 & & Pdr-Sicilia Futura & 6 & \\
\hline & Unione Consumatori & 0 & & Alternativa Popolare & 4,2 & \\
\hline & Tot. Coalizione & 30,4 & & Tot. Coalizione & 25,4 & -5 \\
\hline \multirow[t]{7}{*}{ Musumeci } & & 25,7 & Musumeci & & 39,8 & $-1,3$ \\
\hline & Pdl & 12,9 & & $\mathrm{Fi}$ & 16,3 & \\
\hline & Pid-Cantiere popolare & 5,9 & & $\begin{array}{l}\text { Alleanza per la Sicilia/Nello Mu- } \\
\text { sumeci Pres./Fratelli d'Italia/Noi } \\
\text { con Salvini }\end{array}$ & 5,6 & \\
\hline & Musumeci Presidente & 5,6 & & $\mathrm{Udc}$ & 7 & \\
\hline & Alleanza di centro & 0,3 & & DiventeràBellissima & 6 & \\
\hline & & & & $\begin{array}{l}\text { Idea Sicilia/Popolari e Autono- } \\
\text { misti }\end{array}$ & 7,1 & \\
\hline & Tot. Coalizione & 24,7 & & & & \\
\hline \multirow[t]{6}{*}{ Miccichè } & & 15,4 & & & & \\
\hline & Mpa-Ns & 9,5 & & & & \\
\hline & Grande Sud Miccichè & 6 & & & & \\
\hline & Futuro e Libertà per l'Italia & 4,4 & & & & \\
\hline & Pensiero ed azione & 0,1 & & & & \\
\hline & Tot. Coalizione & 20 & & Tot. Coalizione & 42 & $-2,7$ \\
\hline \multirow[t]{4}{*}{ Marano } & & 6,1 & Fava & & 6,1 & 0 \\
\hline & Idv & 3,5 & & $\begin{array}{l}\text { Cento Passi per la Sicilia/Fava } \\
\text { Presidente }\end{array}$ & 5,2 & \\
\hline & Claudio Fava Pres./Sel/Verdi & 3,1 & & & & \\
\hline & Tot. Coalizione & 6,6 & & Tot. Coalizione & 5,2 & $-1,4$ \\
\hline \multirow[t]{2}{*}{ Altri candidati } & & 4,2 & La Rosa & & 0,7 & $-3,5$ \\
\hline & Altre liste & 3,4 & & Siciliani Liberi & 0,6 & $-2,8$ \\
\hline
\end{tabular}

Quanto al rapporto (elettoralmente parlando) tra i candidati alla presidenza della Regione e le coalizioni di riferimento, il primo dato interessante è costituito dall'ottimo risultato del candidato del M5s Cancelleri $(34,6 \%)$ che è di quasi 10 punti percentuali sopra il risultato della lista di riferimento $(26,7 \%)$ e ad un più $16,4 \%$ rispetto al risultato ottenuto nel 2012 , in linea con le europee del $2014(26,3 \%)$ ma al di sotto delle nazionali del 2013 (33,6\%). Il vero vincitore morale è lui.

A questo dato si aggiunge quello relativo alla coalizione di centrodestra, uscita vincitrice dalle urne, attraversata da una situazione interna molto più controversa di quanto non appaia. La coalizione a sostegno di Musumeci si è presentata unita rispetto alle precedenti elezioni del 2012 quando il centrodestra si presentò spaccato e con due candidati: lo stesso Musumeci e Gianfranco Miccichè. Ebbene, nel 2017 la coalizione di centrodestra ottiene complessivamente 50 mila voti in meno rispetto al 2012 nonostante della coalizione di centrodestra è rientrata a far parte l'Udc. Complessivamente il candidato Musumeci ottiene più voti della sua coalizione di riferimento capitalizzando il vantaggio di avere avuto più liste (e conseguentemente più candidati) a suo sostegno. In sostanza, la coalizione mostra una maggiore capacità aggregativa sottolineata dalla crescita al suo interno della componente più radicale grazie al sostegno dalla Lega e da Fratelli d'Italia.

All'interno della coalizione di centrosinistra, invece, si giocano tante partite contemporaneamente. Il candidato Micari ottiene un risultato elettorale molto deludente sia rispetto alla coalizione di riferimento $(-6,8 \%)$ sia rispetto al suo predecessore Crocetta nel $2012(-11,9 \%)$. La coalizione complessivamente mantiene le perdite grazie all'apporto delle due liste del Pdr-Sicilia e di Alternativa popolare che hanno compensato, per così dire, i consensi ottenuti nel 2012 dall'Udc e dalla lista civica il Megafono. Anche il Pd, alle prese con le divisioni interne, mantiene il consenso ottenuto nel 2012 rispetto al quale perde solo lo 0,4\%. L'unico 
elemento di criticità è rappresentato dal fatto che soltanto due delle liste della coalizione (Pd e Pdr-Sicilia Futura) riescono a superare lo sbarramento e, dunque, ad ottenere seggi all'Ars determinando una situazione di dis-rappresentatività dell'intera coalizione.

Discorso a parte merita la sinistra radicale rappresentata dal candidato Claudio Fava e dalla lista Cento Passi per la Sicilia che mantiene pressoché gli stessi consensi del 2012 fermandosi al $6,1 \%$. Sotto questo punto di vista, l'unico elemento di novità è rappresentato dal fatto che nonostante la lista Cento Passi per la Sicilia abbia ottenuto meno consensi rispetto alla coalizione del 2012 riesce ad ottenere lo storico risultato di conquistare un seggio all'Ars dopo 16 anni. Se si sommano i voti ottenuti dalla coalizione di centrosinistra con i voti ottenuti dalla sinistra radicale complessivamente la sinistra indietreggia sia se prendiamo a riferimento le liste regionali (dunque i candidati a presidente della Regione) sia se sommiamo le percentuali delle liste di riferimento.

Una questione rilevante, inoltre, è quella che riguarda la forte presenza del voto personale che ha determinato travasi di voti da una parte all'altra, tratto distintivo dell'elettorato siciliano. Gli analisti dei flussi elettorali hanno evidenziato travasi di consensi nelle più diverse direzioni: dal centrosinistra al centrodestra e viceversa. Il travaso di voti non ha risparmiato neanche il M5s tanto da far pensare che questo fenomeno sia più legato a situazioni politiche locali e contingenti più che ad un cambiamento nell'orientamento politico in generale, o di valutazione dell'offerta politica. Il dato elettorale più significativo che emerge dall'osservazione dei flussi è lo "sparigliamento" del bottino elettorale che aveva portato all'elezione del governatore Crocetta nel 2012 (Vignati, 2017). Gli elettori che avevano aderito, attraverso il voto, a quel progetto politico nel 2012 si sono riposizionati nelle più diverse direzioni. Pochi di questi consensi nel 2017 si sono riversati sul candidato di centrosinistra Micari - che a sua volta ha captato molti degli elettori che nel 2012 avevano sostenuto il candidato di centrodestra Miccichè -, buona parte è andata a sostegno del candidato dei cinquestelle Cancelleri e il candidato di centrodestra Musumeci, mentre la restante parte ha scelto l'astensione.

TAB. 9 - Voti validi solo per le liste reg. per provincia 2012 e differenza con le elezioni regionali in Sicilia 2012; val. \%

\begin{tabular}{|c|c|c|c|}
\hline \multicolumn{4}{|c|}{ Voti validi solo per le liste regionali per provincia 2012 e differenza con le elezioni regionali in Sicilia 2012; val. \%. } \\
\hline \\
\cline { 2 - 4 } & $\begin{array}{c}\text { Regionali } \\
2012\end{array}$ & $\begin{array}{c}\text { Regionali } \\
2017\end{array}$ & $\begin{array}{c}\text { Diff. } \\
2017 / 2012\end{array}$ \\
& $\begin{array}{c}\text { voti solo } \\
\text { candidati } \\
\text { presidente }\end{array}$ & $\begin{array}{c}\text { voti solo } \\
\text { candidati } \\
\text { presidente }\end{array}$ & \\
\hline Siracusa & 2,4 & 4,1 & 1,8 \\
\hline Catania & 2,9 & 4,2 & 1,4 \\
\hline Palermo & 2,3 & 3,5 & 1,2 \\
\hline Enna & 2,2 & 3,3 & 1,1 \\
\hline Trapani & 2,0 & 3,0 & 1,0 \\
\hline Messina & 1,8 & 2,7 & 0,9 \\
\hline Caltanissetta & 2,5 & 3,3 & 0,8 \\
\hline Ragusa & 3,6 & 4,3 & 0,7 \\
\hline Agrigento & 1,4 & 2,0 & 0,7 \\
\hline Sicilia & 2,3 & 3,4 & 1,0 \\
\hline
\end{tabular}

Infine il dato sul voto esclusivamente dato alle liste regionali, dunque ai candidati alla presidenza della Regione. Come si vede non si registra un ricorso massiccio a questa modalità di espressione del voto. Si registra un aumento generalizzato delle percentuali in tutte le circoscrizioni elettorali provinciali ma non superano il differenziale di 1,8 punti rispetto alle elezioni regionali del 2012, è questo il caso della provincia di Siracusa. La media percentuale regionale è complessivamente di 3,4 punti anche se la differenza complessiva rispetto al 2012 non supera il punto.

Discorso a parte invece merita il ricorso all'altra modalità elettorale possibile, vale a dire, quella del voto disgiunto. In questo caso molti indicatori tra cui i dati che abbiamo ampiamente analizzato in precedenza ci dicono che gli elettori siciliani ne hanno fatto largo uso. Tra le motivazioni di tale comportamento elettorale un mix di fattori esplicativi su alcuni dei quali ci siamo già soffermati - come la complessità dell'offerta politica - ed altri su cui ri- 
fletteremo più avanti.

\section{La personalizzazione del rapporto di rappresentanza}

Una delle costanti della storia elettorale siciliana è costituita dall'elevato grado di personalizzazione della politica che si presenta sotto due possibili forme: la macro-personalizzazione e la micro-personalizzazione (cfr., tra gli altri, Calise 2000 e 2010; Bolgherini e Musella 2006). La prima forma vede come protagonisti i candidati alla presidenza, in un rapporto del tipo uno-a-molti, sostanzialmente indiretto ${ }^{40}$, la seconda sottolinea, invece, il ruolo dei candidati consigliere nella mobilitazione e nell'organizzazione del consenso, a fare la differenza sono in questo caso le caratteristiche personali e le reti di relazioni con gli elettori dei candidati alla carica di consigliere, in un rapporto del tipo uno-ad-uno. Naturalmente il primo fenomeno non esclude in radice il secondo (e viceversa), anche se esiste un trade off tra i due. Diversi indicatori segnalano l'evoluzione e l'intensità del processo di macropersonalizzazione, tra gli altri: l'indice di personalizzazione, ovvero il rapporto fra voti raccolti dal presidente e voti destinati alla coalizione; e la presenza e il peso delle cosiddette liste del presidente (in alcuni casi veri e propri partiti o movimenti "personali", costruiti attorno a leader locali). Gli indici di personalizzazione che compaiono nella tabella 10 ci consentono di misurare se e quanto in termini percentuali il consenso al presidente ecceda quello rivolto alle liste che lo sostengono per le legislature dalla XIII legislatura (2001-06) alla XVII (2017-...). I dati sono facilmente interpretabili: un valore pari a uno indica che le preferenze al candidato presidente coincidono con quelle attribuite alla sua coalizione di riferimento, mentre valori superiori all'unità indicano che una quota di elettori ha scelto di votare per il candidato presidente senza indicare alcun partito o lista. Ad es., un indice dell'1,10 indica che il candidato presidente gode di un consenso del $10 \%$ superiore a quello ottenuto dai partiti della sua coalizione, viceversa un indice dello 0,90 indica che la coalizione ha raggiunto voti superiori di 10 punti percentuali rispetto al candidato presidente. Un primo dato che emerge dall'analisi della tabella è una tendenza generalizzata a valori dell'indice superiori a uno, ciò significa che, salvo rari casi (Cuffaro nel 2001 e 2006 e Micari nel 2017), i voti attribuiti al candidato presidente sono sempre maggiori rispetto a quelli della coalizione di riferimento (il dato medio per tutte le 5 legislature è 1,10 ). Una seconda regolarità è data dal fatto che gli indici di personalizzazione sono relativamente più alti nelle coalizioni di centrosinistra (in media 1,10) e nel Movimento 5 Stelle (in media 1,35) rispetto alle coalizioni di centrodestra. Le prime sono quelle, cioè, che esprimono maggiormente un consenso di tipo autonomo nei confronti dei candidati alla presidenza. Una riflessione a parte merita il dato del M5s: in questo caso, l'indice di personalizzazione, in media pari a 1,35, indica che molti elettori preferiscono votare il candidato alla presidenza piuttosto che la lista del Movimento.

TAB. 10 - Indice di personalizzazione nella Regione Sicilia (2001-2017)

\begin{tabular}{|c|c|c|c|c|c|c|c|c|c|c|c|c|}
\hline & \multicolumn{2}{|c|}{2001} & \multicolumn{2}{|c|}{2006} & \multicolumn{2}{|c|}{2008} & \multicolumn{3}{|c|}{2012} & \multicolumn{3}{|c|}{2017} \\
\hline & $\begin{array}{c}C S \\
\text { Orlando }\end{array}$ & $\begin{array}{c}C D \\
\text { Cuffaro }\end{array}$ & $\begin{array}{c}C S \\
\text { Borsellino }\end{array}$ & $\begin{array}{c}C D \\
\text { Cuffaro }\end{array}$ & $\begin{array}{c}C S \\
\text { Finocchiaro }\end{array}$ & $\begin{array}{c}C D \\
\text { Lombardo }\end{array}$ & $\begin{array}{c}C S \\
\text { Crocetta }\end{array}$ & $\begin{array}{c}C D \\
\text { Musumeci }\end{array}$ & $\begin{array}{c}\text { M5S } \\
\text { Cancelleri }\end{array}$ & $\begin{array}{c}C S \\
\text { Micari }\end{array}$ & $\begin{array}{c}C D \\
\text { Musumeci }\end{array}$ & $\begin{array}{c}\text { M5S } \\
\text { Cancelleri }\end{array}$ \\
\hline Sic. & 1,29 & 0,97 & 1,22 & 0,91 & 1,12 & 1,02 & 1,06 & 1,10 & 1,29 & 0,80 & 1,03 & 1,41 \\
\hline
\end{tabular}

Fonte: Elaborazione propria da dati ufficiali Ufficio Elettorale Regione Sicilia.

Per ogni tornata elettorale, in grassetto, l'indice di personalizzazione del candidato vincente.

L'indice di personalizzazione è pari al rapporto tra i voti espressi solo per il candidato alla presidenza e il totale dei voti ai partiti della coalizione che sosteneva quel candidato.

Già utilizzate con successo e in modo crescente nelle elezioni amministrative - siamo al secondo indicatore - le liste identificate dal nome del candidato alla presidenza (le cosiddette liste del presidente) hanno fatto la loro comparsa anche nelle elezioni regionali siciliane, un ulteriore segnale della rilevanza che i candidati alla presidenza hanno assunto nella competizione elettorale. Oltre a ricondurre maggiormente la contesa alla sua dimensione regionale,

${ }^{40}$ Sul tema della presidenzializzazione della politica, una delle espressioni della macropersonalizzazione, si veda in generale Poguntke e Webb 2005. 
la presenza delle liste legate al candidato presidente punta a valorizzare la propria azione di governo per gli uscenti ma, soprattutto, a segnalare la loro (relativa) autonomia dai partiti. È a partire dalle elezioni regionali del 2006 che i principali candidati presidente hanno presentato nella competizione proporzionale una lista a lui/lei direttamente collegata (si veda tab. 11): il loro rendimento ha oscillato, in media, intorno al 4,3\%. A superare la soglia di sbarramento prevista dal 2005 dalla legge elettorale siciliana sono state: la lista L'Aquilone Lista del Presidente di Cuffaro nel 2006, le liste Nello Musumeci Presidente, Movimento Politico Crocetta Presidente e Grande Sud Miccichè nel 2012 e la lista Nello Musumeci DiventeràBellissima nel 2017.

TAB. 11 - Liste del presidente, elezioni regionali (2006-2017)

\begin{tabular}{|c|c|c|c|}
\hline & Liste personali & Voti $\%$ & Seggi \\
\hline \multirow[t]{3}{*}{2006} & L'Aquilone Lista del Presidente - Cuffaro & 5,7 & 6 \\
\hline & Rita Il mio impegno per la Sicilia - Borsellino & 4,8 & - \\
\hline & Nello Musumeci Alleanza Siciliana - Musumeci & 2,4 & - \\
\hline \multirow[t]{3}{*}{2008} & Lombardo Presidente Sicilia Forte e Libera - Lombardo & 4,5 & - \\
\hline & Anna Finocchiaro Presidente per la Sicilia - Finocchiaro & 3,1 & - \\
\hline & Amici di Beppe Grillo. Con Sonia Alfano Presidente - Alfano & 1,7 & - \\
\hline \multirow[t]{4}{*}{2012} & Nello Musumeci Presidente - Musumeci & 5,6 & 4 \\
\hline & Movimento Politico Crocetta Presidente - Crocetta & 6,2 & 5 \\
\hline & Grande Sud Miccichè - Miccichè & 6,0 & 5 \\
\hline & Claudio Fava Presidente Fds Sel Verdi - Fava & 3,1 & - \\
\hline \multirow[t]{2}{*}{2017} & Nello Musumeci DiventeràBellissima - Per la Sicilia - Musumeci & 6,0 & 4 \\
\hline & Arcipelago Sicilia Movimento dei Territori Micari Presidente - Micari & 2,2 & - \\
\hline
\end{tabular}

Nel caso siciliano, nelle liste del presidente confluiscono spesso o sono diretta emanazione di vere e proprie "liste personali" o movimenti che ruotano attorno a leader locali. Così, nel 2006, Nello Musumeci rifiuta di appoggiare la candidatura del presidente uscente Salvatore Cuffaro e si candida alla presidenza della Regione siciliana a capo del suo movimento, Alleanza Siciliana, un movimento autonomista di destra a carattere regionale; nel 2008, non possiamo non accennare al fatto che, oltre alla lista Lombardo Presidente Sicilia Forte e Libera, Raffaele Lombardo contava sul proprio movimento, il Movimento per le Autonomie Mpa, il partito autonomista di Lombardo otterrà il 14\% (e 15 seggi all'Ars) - terzo partito nell'isola dopo il Pdl e il Pd; nel 2012, è il candidato presidente del centrosinistra ad essere espressione del proprio movimento, il Movimento Politico Crocetta Presidente, la lista otterrà il $6,2 \%$ dei voti e 5 deputati all'Ars e concorrerà in maniera decisiva al successo di Rosario Crocetta; infine, nel 2017, Nello Musumeci è, all'inizio, il candidato ufficiale del suo movimento DiventeràBellissima, che lo propone alla presidenza della Regione, candidatura che incontrerà solo dopo il sostegno di FdI, Noi con Salvini, Udc, Fi ed autonomisti.

La micro-personalizzazione trova riscontro empirico, invece, nell'elevato uso del voto di preferenza, in genere considerato come indice della forte personalizzazione della cultura politica siciliana, se non addirittura della prevalenza di legami semplicemente clientelari tra cittadini, ceto politico e istituzioni. Il tasso di preferenza in questa tornata si abbassa di più di 5 punti percentuali, attestandosi al 77,8\%, rispetto alle elezioni del 2012 (quando il tasso di preferenza era pari all' $83,5 \%$ ), tale calo è dovuto solo in parte alla differente consistenza elettorale del M5s, passato dal 14,9\% al 26,7\%, con un tasso di preferenza che si mantiene però basso rispetto alle altre forze politiche: in assenza del M5s, il tasso di preferenza avrebbe raggiunto l' $85 \%$ nel 2017 (vs. 1'89,5\% nel 2012).

Se vogliamo, però, delineare il fenomeno meglio di quanto non appaia dal mero riferimento all'indice aggregato, dobbiamo dare una adeguata enfasi al fatto che il fenomeno assume toni diversi non solo in relazione alle diverse forze politiche (si veda fig. 1), ma anche con riferimento alla distribuzione interna all'isola (si veda tab. 12). 
Fig. 1-L'uso della preferenza: differenziazioni partitiche

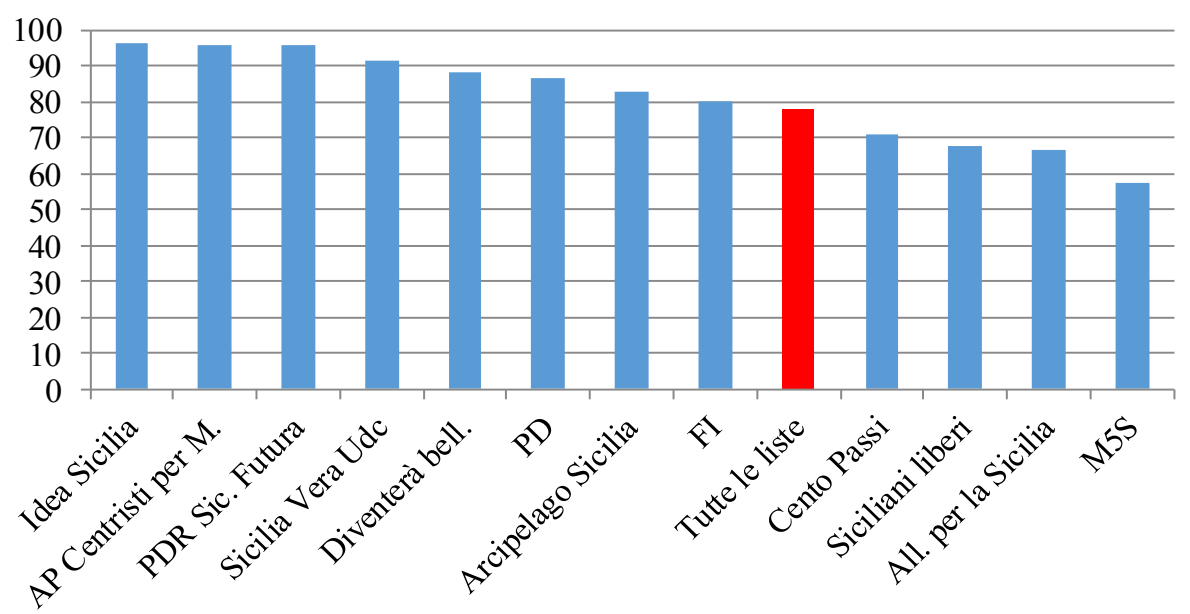

I tassi di preferenza sono calcolati dal rapporto percentuale fra voti di preferenza espressi e voti di lista.

Come si vede dalla figura 1, Idea Sicilia, AP Centristi per Micari, Pdr Sicilia Futura e Sicilia Vera Udc, ovvero le 4 liste post-democristiane, risultano quelle maggiormente in grado di attrarre voti di preferenza, se si esclude Sicilia Vera Udc che si ferma al 91,6\%, le altre tre liste post-democristiane superano addirittura il 95\% (Idea Sicilia Popolari e Autonomisti è al 96,5\%; AP Centristi per Micari è al 96,1\%; Pdr Sicilia Futura al 95,6\%). Segue all'88,4\% il movimento DiventeràBellissima di Nello Musumeci. Il Pd arriva all' $86,8 \%$, superando tanto la lista Arcipelago Sicilia (83\%) quanto, per la prima volta, la stessa Fi (80\%). Sotto la media le liste Cento Passi per la Sicilia (70,9\%), Siciliani Liberi (67,5\%) e Alleanza per la Sicilia $(66,6 \%)$. Ma a staccarsi in modo significativo è soprattutto il M5s, per il quale il tasso di preferenza è pari al 57,7\%; sebbene, anche il M5s continui il suo processo di "normalizzazione": il suo tasso di preferenza passa dal 37,7\% nel 2008 (con la lista Amici di Beppe Grillo) al 49,6\% nel 2012, al 57,7\% delle ultime elezioni regionali. Il dato medio peraltro nasconde una forte differenziazione territoriale del Movimento, il tasso di preferenza va da poco più del $50 \%$ nelle province di Catania $(51,6 \%$ ) e Palermo (52,3\%), a tassi che sfiorano il $70 \%$ nelle province di Agrigento $(69,8 \%)$ e Caltanissetta $(68,2 \%)$, la provincia di Giancarlo Cancelleri.

La tab. 12 riporta i valori del tasso di preferenza registrati nell'intera Regione e nelle sue nove province, fornendoci un quadro della distintività territoriale nell'uso del voto di preferenza dal 2001 al 2017. Il campo di variazione viene delineato dalla polarizzazione tra la provincia di Agrigento, la media del periodo è 85,8 , una realtà dove la personalizzazione del voto è sempre stata estesa, e la provincia di Ragusa, la media del periodo è 74,2 , una realtà dove il rapporto individualistico con la politica, ancorché presente, ha assunto da sempre connotazioni diverse, meno marcate rispetto al resto della regione.

TAB. 12 - Tassi di preferenza per provincia nelle elezioni regionali siciliane (2001-2017)

\begin{tabular}{|c|c|c|c|c|c|}
\hline & 2001 & 2006 & 2008 & 2012 & 2017 \\
\hline Agrigento & 88,2 & 90,2 & 77,7 & 88,2 & 84,5 \\
\hline Caltanissetta & 84,4 & 86,9 & 71,1 & 84,4 & 80,4 \\
\hline Catania & 82,6 & 85,9 & 70,2 & 83,6 & 74,9 \\
\hline Enna & 76,8 & 85,4 & 63,9 & 80,8 & 74,6 \\
\hline Messina & 84,9 & 87,2 & 74,2 & 74,2 & 82,1 \\
\hline Palermo & 82,9 & 85,6 & 71,5 & 82,6 & 75,9 \\
\hline Ragusa & 75,3 & 82,1 & 65,5 & 75,2 & 73,0 \\
\hline Siracusa & 83,5 & 86,1 & 68,0 & 82,1 & 76,0 \\
\hline Trapani & 84,0 & 86,1 & 69,1 & 83,6 & 79,9 \\
\hline Sicilia & 83,2 & 86,3 & 71,0 & 83,5 & 77,8 \\
\hline
\end{tabular}


Il voto di preferenza sta ad indicare nei rapporti tra rappresentanti e rappresentati il salto della mediazione dei partiti in quanto attori collettivi del processo politico: "l'alta personalizzazione del voto contraddistingue, dunque, i rapporti tra società civile e sistema dei partiti, e assume talvolta $\mathrm{i}$ toni di un esasperato individualismo che implica che il voto alla singola persona preceda e sovrasti quello al partito" (D'Amico 1993, 211). Questo risulta tanto più vero per i cosiddetti campioni delle preferenze, ovvero candidati che detengono un rilevante pacchetto di voti che viene mantenuto nel tempo e spesso spostato indipendentemente dalle scelte partitiche o coalizionali (Emanuele e Marino 2016).

Nella tabella 13 abbiamo indicato i campioni delle preferenze che nelle elezioni regionali del 2017 hanno superato le 10 mila preferenze. È evidente, anzitutto, la trasversalità politica di questi candidati ( 6 sono del Pd, 5 del M5s, 5 di Fi, 3 del Pdr Sicilia Futura). In secondo luogo, risalta la presenza di ben 5 campioni delle preferenze del M5s, a conferma della normalizzazione alle dinamiche di voto della Regione, ad essere riconoscibile non è più solo la lista ma anche i singoli candidati del Movimento, che in gran parte dei casi 5 anni fa erano poco o nulla conosciuti sul territorio.

TAB. 13 - I campioni delle preferenze nelle elezioni regionali 2017, preferenze $>10.000$ [14 su 19 ricandidati e rieletti]

\begin{tabular}{|c|c|c|c|}
\hline & Lista & Circoscrizione & Preferenze \\
\hline Sammartino Luca & $\mathrm{PD}$ & $\mathrm{CT}$ & 32.242 \\
\hline Zito Stefano & M5S & SR & 18.008 \\
\hline Genovese Luigi & FI & $\mathrm{ME}$ & 17.359 \\
\hline $\begin{array}{l}\text { Mangiacavallo Mat- } \\
\text { teo }\end{array}$ & M5S & $\mathrm{AG}$ & 14.973 \\
\hline Barbagallo Anthony & PD & $\mathrm{CT}$ & 14.228 \\
\hline Tamajo Edmondo & PDR & PA & 13.984 \\
\hline $\begin{array}{l}\text { Calderone Tomma- } \\
\text { so }\end{array}$ & FI & ME & 13.517 \\
\hline Cancelleri Giovanni & M5S & $\mathrm{CL}$ & 12.434 \\
\hline Falcone Marco & FI & $\mathrm{CT}$ & 12.045 \\
\hline Foti Angela & M5S & $\mathrm{CT}$ & 11.593 \\
\hline Villari Angelo & PD & $\mathrm{CT}$ & 11.256 \\
\hline $\begin{array}{l}\text { De Domenico Fran- } \\
\text { cesco }\end{array}$ & PD & ME & 11.224 \\
\hline Germanà Antonino & FI & $\mathrm{ME}$ & 11.046 \\
\hline D'Agostino Nicola & PDR & $\mathrm{CT}$ & 10.909 \\
\hline $\begin{array}{l}\text { Guicciardi Baldas- } \\
\text { sare }\end{array}$ & PD & $\mathrm{TP}$ & 10.891 \\
\hline Ciancio Gianina & M5S & $\mathrm{CT}$ & 10.584 \\
\hline Tranchida Giacomo & PD & $\mathrm{TP}$ & 10.578 \\
\hline Picciolo Giuseppe & PDR & ME & 10.242 \\
\hline Papale Alfio & FI & $\mathrm{CT}$ & 10.159 \\
\hline Totale & - & - & 257.272 \\
\hline
\end{tabular}

Il primo tra i campioni delle preferenze è un esponente del Pd, Luca Sammartino, 32 anni e 32 mila voti raccolti nella sua provincia, il miglior risultato di sempre nella storia dell'Ars (il record apparteneva al palermitano Antonello Antinoro eletto nelle file dell'Udc, nel 2006, con 30.202 preferenze). Un record persino in comparazione con gli altri candidati: il secondo classificato, Stefano Zito, del Movimento 5 Stelle arriva a 18.008 preferenze, Luigi Genovese, di Fi, famoso per essere il "figlio di Francantonio" - ras delle preferenze a Messina, condannato in primo grado a 11 anni di reclusione nel processo messinese "Corsi d'Oro" sullo scandalo della formazione professionale regionale - a 17.359, staccati entrambi di quasi 15 mila preferenze rispetto a Sammartino ${ }^{41}$.

${ }^{41}$ Medico odontoiatra, eletto all'Ars nel 2012 con l'Udc con 12.576 preferenze, passato poi ad Articolo 4 (movimento creato da un altro centrista ed ex Mpa, Lino Leanza) e, infine, approdato al Pd grazie al legame con il renziano Davide Faraone (cfr. Chi è Luca Sammartino, nuovo "vasa vasa" re del consenso in Sicilia, R. Ferrazza, Il Sole 24 Ore, 8 novembre 2017). 


\section{La nuova Ars e la nuova Giunta}

La coalizione di centrodestra porta all'Assemblea Regionale 29 deputati, a cui si aggiungono i 7 del listino (compreso il nuovo presidente Musumeci), all'opposizione il primo gruppo è quello del Movimento 5 Stelle (20 deputati, 19 ottenuti col sistema proporzionale più un seggio che va di diritto al candidato presidente secondo classificato, Giancarlo Cancelleri nel 2012 i deputati del M5s erano 15). Seguono il Pd con 11 deputati (nel 2012 erano 14), Pdr Sicilia futura con 2 e la lista Cento Passi per la Sicilia con il seggio conquistato da un altro candidato presidente, il candidato della sinistra, Claudio Fava (si veda fig. 2).

Fig. 2 - La nuova Assemblea Regionale Siciliana

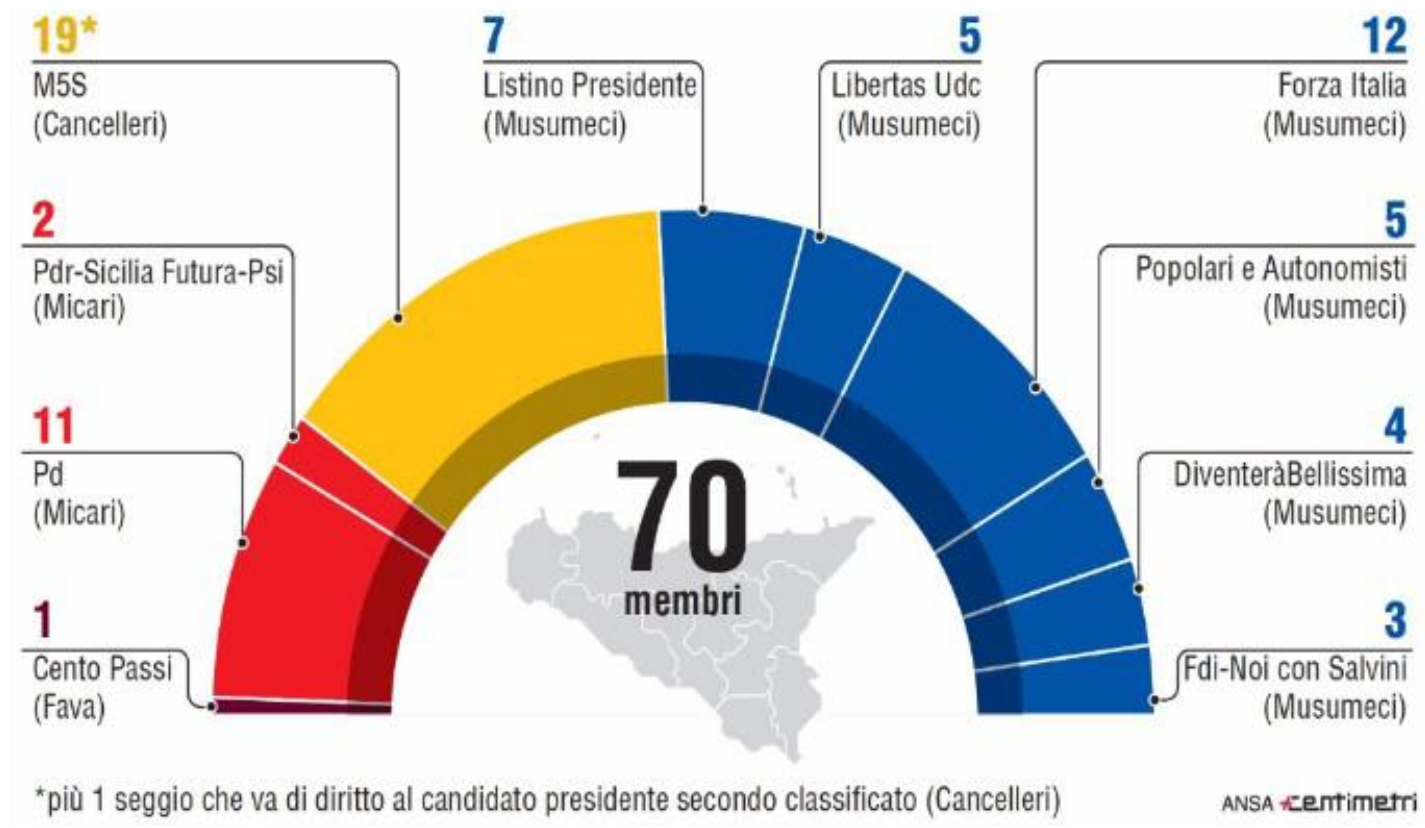

Il nuovo Presidente, Nello Musumeci, potrà contare su una maggioranza risicata: la metà più uno dei 70 deputati di cui è formata l'Ars, ma cinque anni fa, Rosario Crocetta iniziò il suo mandato con appena 39 deputati (su 90), anche se negli anni successivi diversi deputati sono stati pronti - per citare Ennio Flaiano - a correre in aiuto del vincitore. Ma chi sono i nuovi eletti all'Ars?

Un primo aspetto che conviene prendere in considerazione riguarda il tasso di ricambio dell'Assemblea: su 70 membri ben 36 risultano ri-eletti (il tasso di ricambio è pari al 48,6\%). Fra i 34 neo-eletti, peraltro, solo alcuni sono al primo mandato perché in diversi casi si tratta di un ritorno all'Ars ${ }^{42}$ : se si tiene conto di questi ritorni il tasso di ricambio si abbassa ulteriormente (a meno del 40\%!). La forza dell'incumbency (il vantaggio della visibilità e delle risorse organizzative di cui godono gli eletti in carica) riduce, ancora, considerevolmente i margini per l'entrata di nuovi competitori.

Un altro indicatore di chiusura/apertura della classe politica riguarda la presenza delle donne. La Sicilia è stata tradizionalmente una regione inospitale per le donne: quando sono state presenti in Assemblea non hanno mai superato il limite massimo di 4 (su 90 deputati). Da questo punto di vista le ultime due legislature rappresentano una assoluta novità nell'Ars:

42 Ritornano all'Ars: l'ex presidente dell'Ars Gianfranco Miccichè, plenipotenziario di Silvio Berlusconi in Sicilia (e architetto dell'accordo con Nello Musumeci), Giuseppa Savarino, deputata all'Ars nella XIII e XIV legislatura, Eleonora Lo Curto deputata nella XIII legislatura e poi eurodeputata, Cateno De Luca, deputato nella XIV e XV, Caronia Maria Anna ed Alessandro Aricò entrambi deputati nella XV legislatura, lo stesso Roberto Lagalla, già assessore regionale alla sanità nel governo Cuffaro (2006-08). 
nelle elezioni del 2012 vengono elette ben 15 donne (su 90, pari al 16,7\%) ${ }^{43}$, nel 2017 le donne diventano addirittura 16 , ma in proporzione risultano in numero ancora maggiore in quanto la nuova Ars è formata ora da 70 membri (il tasso di presenza femminile sale, infatti, al 22,9\%). Il 50\% delle deputate sono state elette con il M5s, ben otto su 16 (il $40 \%$ degli eletti del M5s), il centrodestra conta 7 donne su 36 eletti ( 3 Fi, 2 Udc, 1 FdI, 1 DiventeràBellissima), il centrosinistra solo 1 donna su 14 eletti (1 Pd).

Questa legislatura verrà, poi, ricordata anche per un altro record, un record siciliano niente affatto invidiabile; all'insediamento dell'Assemblea si sono presentati 5 neo deputati già sotto indagine: Cateno De Luca, leader di Sicilia Vera, arrestato per evasione fiscale ancora prima della proclamazione (gli arresti domiciliari verranno poi revocati); Luigi Genovese e Riccardo Savona di Fi, indagati il primo per evasione fiscale e riciclaggio, il secondo per truffa e appropriazione indebita; Tony Rizzotto, primo deputato leghista all'Ars, accusato di appropriazione indebita aggravata; Edy Tamajo di Sicilia Futura accusato di compravendita di voti ${ }^{44}$.

Quanto, infine, alla formazione della giunta, sono serviti venti giorni di gestazione per la nascita della nuova giunta ${ }^{45}$, il presidente Musumeci ha così sciolto gli ultimi nodi affidando le deleghe ai suoi 12 assessori (tra cui solo due donne). Nella scelta Musumeci ha ripartito i posti in base alle preferenze ottenute alle urne dai partiti che lo hanno sostenuto, secondo la "formula del tre", ad ogni partito un assessorato ogni 3\% riportato alle urne - un criterio tutto sommato "semplice e proporzionale". E ciò partendo dai tre nomi che Nello Musumeci aveva già indicato come suoi potenziali assessori: l'avvocato Gaetano Armao, vice presidente in pectore e assessore all'economia; l'ex rettore dell'Università di Palermo Roberto Lagalla all'istruzione e formazione professionale; Vittorio Sgarbi, ai beni culturali ("a tempo", per impegnarsi poi nelle elezioni politiche $)^{46}$ - si veda tab. 14 .

${ }^{43}$ Nella XVI legislatura, inoltre, su 47 assessori regionali che si sono alternati nelle giunte Crocetta, 18 sono state donne (il 38,3\%) - nella prima giunta Crocetta le donne erano addirittura 7 su 12 (il $58,3 \%$ ), impiegate in tutti i settori dell'amministrazione regionale dalla sanità con Lucia Borsellino alla vicepresidenza con Mariella Lo Bello.

${ }^{44}$ Cfr. Regione, l'avvio choc della legislatura a Palazzo dei Normanni, F. Russello, La Sicilia, 15 novembre 2017; Il record siciliano, Repubblica.it, 8 novembre 2017. Per i singoli casi si vedano Elezioni Sicilia, Messina: arrestato per evasione fiscale il neo-deputato regionale De Luca (Udc), Repubblica.it Palermo, 8 novembre 2017; Fondi esteri per 16 milioni”: indagato a Messina Luigi Genovese, neo eletto all'Ars, C. Marasca, CdS-Corriere del Mezzogiorno, 23 novembre 2017; Regione, indagato un altro deputato: è Riccardo Savona, Repubblica.it Palermo, 21 novembre 2017; Appropriazione indebita: indagato Tony Rizzotto, Giornale di Sicilia, 7 dicembre 2017; Sicilia, indagato neo eletto Tamajo: è accusato di compravendita voti, Il Sole 24 Ore, 11 novembre 2017.

${ }^{45}$ Musumeci, cui era stata rimproverata una scarsa attenzione nella formazione delle liste, già tre giorni dopo la sua elezione, aveva detto: "Se nella fase della composizione delle liste qualcuno non ha seguito i miei reiterati inviti alla prudenza, tutte le scelte che dipenderanno da me invece saranno improntate a questo criterio, a cominciare dalla formazione della giunta".

${ }^{46}$ Sgarbi candidato dalla coalizione di centrodestra nel collegio uninominale di Acerra perderà nettamente la sfida contro il candidato premier del M5s, Luigi Di Maio (63,4\% vs. 20,4\%), ma verrà comunque eletto alla Camera come capolista del proporzionale nel collegio di Ferrara e Modena. L'11 aprile 2018 viene ufficializzata la nomina del nuovo assessore, Sebastiano Tusa, già dirigente della Regione Siciliana. 
TAB. 14 - Il governo della XVII legislatura (59 ${ }^{\circ}$ governo)

\begin{tabular}{|l|r|}
\hline \multicolumn{2}{|c|}{ Presidente della Regione } \\
\hline \multicolumn{2}{|c|}{ Nello Musumeci } \\
\hline Assessorato agricoltura, sviluppo rurale e pesca mediterranea & Edgardo Bandiera (Fi) \\
\hline Assessorato attività produttive & Bernadette Felice Grasso (Fi) \\
\hline Assessorato autonomie locali e della funzione pubblica & Gaetano Armao (in quota Fi) \\
\hline Assessorato beni culturali e dell'identità siciliana & Vittorio Sgarbi - da aprile 2018 Sebastiano Tusa (in quota Fi) \\
\hline Assessorato economia (e vice presidenza della Regione) & Mariella Ippolito (Idea Sicilia Pop. e Aut.) \\
\hline Assessorato energia e dei servizi di pubblica utilità & Marco Falcone (Fi) \\
\hline Assessorato famiglia, politiche sociali e del lavoro & Roberto Lagalla (Idea Sicilia Pop. e Aut.) \\
\hline Assessorato infrastrutture e mobilità & Ruggero Razza (DiventeràBellissima) \\
\hline Assessorato istruzione e formazione professionale & Salvatore Cordaro (Idea Sicilia Pop. e Aut.) \\
\hline Assessorato salute & Sandro Pappalardo (FdI) \\
\hline Assessorato territorio e ambiente & - da febbraio 2018 Alberto Pierobon (Udc) \\
\hline Assessorato turismo, sport e spettacolo &
\end{tabular}

Gli azzurri sono tre (oltre ad Armao e Sgarbi, in quota Fi): il capogruppo uscente di Fi, il catanese Marco Falcone, alle infrastrutture, Bernadette Felice Grasso alle autonomie locali e il siracusano Edgardo Bandiera all'agricoltura, assessorato di peso per il candidato (non eletto) vicino a Stefania Prestigiacomo.

$\mathrm{Ci}$ sono, poi, due assessori autonomisti: oltre a Lagalla di Idea Sicilia, entrano in giunta Salvatore Cordaro (Cantiere Popolare) e Mariella Ippolito (Mpa), le altre due gambe della lista Idea Sicilia Popolari e Autonomisti: il primo, vice di Musumeci in Commissione regionale Antimafia nella legislatura appena chiusa, al territorio e ambiente; la seconda, alla famiglia e al lavoro.

Due gli assessori Udc: l'ex capogruppo Girolamo Turano, tornato prima delle elezioni, all'Udc di Lorenzo Cesa (dai Centristi di Casini) con delega alle attività produttive $\mathrm{e}$, a sorpresa, Vincenzo Figuccia, passato da pochi mesi da Fi all'Udc, con delega a energia e servizi di pubblica utilità ${ }^{47}$.

A Ruggero Razza di DiventeràBellissima, fedelissimo di Musumeci, va il delicato compito di governare la sanità. Rimane fuori la Lega, la delega al turismo, sport e spettacolo va a Sandro Pappalardo di Fratelli d'Italia. I malumori non hanno tardato a manifestarsi, il deputato della lista Noi con Salvini ha subito lasciato il gruppo parlamentare di FdI per passare al gruppo misto.

Infine, l'Ars ha eletto presidente Gianfranco Miccichè (già presidente Ars nel 2006-2008 con Cuffaro). Miccichè è stato eletto presidente con 39 voti, grazie al fondamentale sostegno dei due deputati di Sicilia Futura e di 4 deputati $\mathrm{Pd}^{48}$.

\section{Conclusioni. Il ritorno del centrodestra tra personalizzazione e polarizzazione}

Il quadro che emerge dalle elezioni regionali siciliane del 5 novembre 2017 conferma, in parte, le aspettative degli osservatori politici che su di esse avevano concentrato l'attenzione. La Sicilia si conferma, ancora una volta, "laboratorio" di sperimentazione di nuovi equilibri politici e di governo in cui si combinano tendenze di lungo periodo e nuovi scenari in una realtà politica che assume forme strane e dove "vecchio" e "nuovo" si confondono. Per dirla con

${ }^{47}$ A sole quattro settimane dall'insediamento Vincenzo Figuccia si dimetterà dopo aver polemizzato con Gianfranco Miccichè per il via libera agli stipendi d'oro (cfr. Stipendi d'oro, Figuccia lascia la giunta Musumeci: "eliminare il tetto lede la dignità dei siciliani”, C. Reale, Repubblica.it Palermo, 27 dicembre 2017; Sicilia, via il tetto agli stipendi d'oro: si dimette per protesta l'assessore Udc Figuccia, Il Messaggero, 27 dicembre 2017): a Figuccia, da tempo in rotta con il commissario di Fi, non era piaciuta l'uscita del neo presidente all'Ars favorevole allo sfondamento del tetto dei burocrati dell'Ars fissato a 240 mila euro e annunciato a poche ore dall'elezione alla presidenza Ars. Il 28 febbraio 2018 viene ufficializzata la nomina del nuovo assessore, Alberto Pierobon, indicato dall'Udc per subentrare al dimissionario Vincenzo Figuccia.

${ }^{48}$ Secondo l'ex capogruppo Pd all'Ars, Antonello Cracolici: "I conti si fanno in fretta, il Pd ha 11 deputati e il nostro candidato ha raccolto 7 voti: ci sono stati 4 utili idioti”" (cfr. Sicilia: Miccichè è il nuovo presidente dell'Ars, Il Sole 24 Ore, 16 dicembre 2017). 
Hirschman (1970), in termini di exit, voice e loyalty, nel quadro politico siciliano, si conferma l'exit, con un tasso di partecipazione che si ferma al 46,7\%, (ri)emerge la voice, con l'ottima performance di un partito-movimento "eccentrico", di protesta o populista il M5s, e c'è un ritorno alla loyalty nei confronti della coalizione "dominante" di centrodestra, una coalizione stavolta coesa (dall'Udc a Fratelli d'Italia) e che garantisce una ritrovata sintonia con gli orientamenti di fondo di un elettorato che è stato, e resta, moderato e conservatore.

In particolare, per quanto riguarda l'exit, come già cinque anni fa, la maggioranza assoluta degli aventi diritto non partecipa: vota il $46,7 \%$, in lieve calo rispetto al $47,4 \%$ del 2012 ; l'unico segnale in controtendenza emerge dalla scomposizione su base provinciale e comunale del dato sulla partecipazione dove si ritrovano valori percentuali al di sopra della media regionale - due province su tutte, Catania $(51,6 \%)$ e Messina $(51,8 \%)$ - così le città capoluogo e i grossi centri i cui valori, generalmente, si attestano sopra il 50\%. Sulla partecipazione al voto e sulla riduzione dell'astensione avevano puntato in campagna elettorale tutti i candidati alle regionali - il M5s, in modo particolare, aveva parlato di riscatto dei delusi come argine all'astensionismo - facendo riferimento ad un bacino elettorale in grado di condizionare il risultato elettorale. Una disaffezione, quella dell'elettorato, preoccupante che non riguarda, peraltro solo la Sicilia basti pensare che la media della partecipazione nelle nove regioni andate al voto nel ciclo elettorale 2014-15 è del 49,6\%, oscillando tra il 57,2\% del Veneto e il 37,7\% dell'Emilia-Romagna, poco sopra quindi al dato siciliano.

L'insoddisfazione e il risentimento degli elettori siciliani non prende soltanto la strada dell'exit dal mercato elettorale ma anche quella della voice, orientandosi verso partiti che più di altri segnano una rottura con il passato. Il M5s si conferma, così, primo partito dell'isola, dopo gli ottimi risultati delle regionali 2012 (14,9\%), delle politiche 2013 (33.6\%), delle europee del $2014(26,3 \%)$, a chiudere il ciclo ci saranno i sorprendenti risultati delle ultime elezioni politiche $(48,7 \%)$. La Sicilia ha periodicamente sentito, sul versante elettorale, l'attrazione per l'offerta politica di protesta: il "milazzismo" degli anni '60, la Rete di Leoluca Orlando e padre Pintacuda sul finire degli anni '80, i movimenti autonomisti come Nuova Sicilia di Bartolo Pellegrino a metà degli anni '90 e il Movimento per l'Autonomia di Raffaele Lombardo intorno al 2005, ma si è trattato sempre di fenomeni di breve durata, intrasistemici. Il dato nuovo riguarda lo strutturarsi dell'offerta politica di protesta, una sfida arrivata stavolta dall'esterno, da non professionisti della politica (Raniolo 2017) ${ }^{49}$.

Quanto al tema del ritorno alla loyalty, dopo la difficile legislatura di Crocetta e della coalizione di centrosinistra, le elezioni regionali siciliane consegnano la vittoria alla coalizione di centrodestra e ad un candidato (Nello Musumeci) che non rappresenta certamente un outsider nel panorama politico siciliano. La sua candidatura (prima) e il successo elettorale (poi) sono frutto di un'abile e paziente strategia elettorale di cui Musumeci è stato certamente il protagonista. Il "successo senza vittoria" di Musumeci ${ }^{50}$ fa tornare la regione in mano alla "coalizione dominante" che, se si esclude la parentesi del governo Crocetta, ha governato ininterrottamente la regione nel corso della Seconda Repubblica, Crocetta vinse, infatti, grazie alle divisioni interne al centrodestra e alla presenza nella sua coalizione dell'Udc che portò in dote un indispensabile 10,8\%. L'operazione riuscita a Crocetta nel 2012 di aggregare una coalizione a trazione centrista - attraverso l'accordo con l'Udc e l'effetto ponte costituito dalla lista del presidente il Megafono, in grado di intercettare i transfughi centristi - non è riuscita a Fabrizio Micari. Per la sinistra e per il centrosinistra si è trattato di una "sonora sconfitta".

Altro dato politico da non sottovalutare è rappresentato dal ruolo e dal peso che hanno avuto, come nelle elezioni del 2012, le liste di centro. Mettendo assieme i voti ottenuti dalle 4 liste di centro (Udc, Popolari e Autonomisti, Ap-Centristi per Micari e Pdr Sicilia Futura) si arriva al $24,3 \%$, quasi mezzo milione di voti $(466.761)$ che si traducono in 12 seggi all'Ars:

${ }^{49}$ La ri-conferma del M5s come primo partito dell'isola sottolinea, inoltre, il passaggio ad un assetto "tripolare" del sistema politico siciliano, con i grillini a contendere il governo dell'isola alla "coalizione dominante".

${ }^{50}$ Quella di Musumeci può essere definita come una "vittoria senza successo" in quanto al successo del candidato non è corrisposto un successo pieno per la coalizione di centro-destra. Il candidato alla presidenza ottiene più voti della coalizione ma, sostanzialmente, perde consensi rispetto al 2012, se si considerano l'insieme dei voti dei candidati e delle liste di riferimento del centro-destra. 
"Numeri che farebbero di questo inesistente ma significativo partito la seconda formazione dell'isola, alle spalle solo del M5s che, nella sua corsa solitaria, ha raccolto il 26,7\%" (Ferrazza 2017, 1). Si tratta di formazioni politiche che come sempre risultano determinanti nella formazione delle maggioranze parlamentari o delle coalizioni politiche, formazioni che in molti casi si coagulano intorno ad esponenti politici locali e che, dunque, coltivano una delle costanti della storia elettorale siciliana, la (micro)personalizzazione della politica. Se il tasso medio di preferenza registrato nel 2017 è più basso di 5 punti percentuali rispetto alla precedente competizione (attestandosi al 77,8\%), sono proprio le liste di centro che mostrano i tassi di preferenza più alti superando addirittura in tre casi su 4 il $95 \%$, all'altro polo il M5s che (nonostante il suo processo di "normalizzazione" 51 alle dinamiche della regione) fa registrare un tasso di preferenza del 57,7\% (comunque in crescita rispetto al 49,6\% del 2012 e al 37,7\% del 2008, quando si presentò con la lista Amici di Beppe Grillo).

Quanto, invece, alla seconda forma di personalizzazione, la personalizzazione in grande, quella dei candidati presidente, bisogna partire dal "successo senza vittoria" del candidato del M5s, Giancarlo Cancelleri, che si è dovuto arrendere, nella competizione con Musumeci, pur avendo ottenuto un ottimo risultato personale $(34,6 \%)$, e pur avendo raddoppiato i voti del 2012. «Noi vincitori morali» è il mantra che accomuna le prime dichiarazioni sui risultati dei portavoce del M5s - il primo è stato l'europarlamentare Ignazio Corrao commentando le prime proiezioni sul voto - definendo «accozzaglia» di liste e di candidati quelle a sostegno del candidato di centrodestra e facendo riferimento al contributo determinante dei candidati "impresentabili" sia per la vittoria del centrodestra sia per il risultato della coalizione di centrosinistra.

La vittoria del candidato Musumeci, che ottiene più voti della sua coalizione di riferimento, è frutto della capacità aggregativa della coalizione di centrodestra rispetto alle altre coalizioni. Viceversa, nella coalizione di centro-sinistra, invece, è emblematica la "scarsa" performance elettorale del candidato Micari che ottiene un risultato negativo sia rispetto alla coalizione di riferimento $(-6,8 \%)$ sia rispetto al suo predecessore Crocetta nel $2012(-11,9 \%)$. Tra i fattori che determinano la scarsa performance del candidato del centro-sinistra: il giudizio negativo dei siciliani sull'amministrazione regionale uscente, la limitata notorietà del candidato di centro-sinistra e l'occasione mancata di una candidatura unitaria con la sinistra radicale.

In sintesi, sembra consolidarsi in Sicilia un sistema politico tripolare a coalizione dominante più che a partito dominante, com'era nel passato. "Coalizione dominante" perché nessun partito riesce a controllare la maggioranza assoluta del mercato elettorale, né tanto meno la maggioranza dei seggi dell'Ars. A cui si aggiunge l'incognita rappresentata dal partito dell'astensione (a cui aderisce un siciliano su due) che potrebbe determinare continui capovolgimenti di fronte dagli esiti imprevedibili sul sistema politico siciliano.

In questo quadro un rischio concreto potrebbe essere rappresentato da un governo della Regione condizionato dai rappresentanti dei partiti elettoralmente minoritari ma che hanno in mano un formidabile potere di ricatto: una condizione di moltiplicazione dei veto players. Questa situazione è aggravata dalla tendenza alla doppia "polarizzazione" a livello di governo e di opposizione (Raniolo, 2017). Se si osserva la composizione politica della coalizione di maggioranza e di opposizione all'Ars si nota una tendenza alla radicalizzazione della rappresentanza politica. Non è da sottovalutare, infatti, il fatto che nella coalizione di centrodestra ha assunto una rilevanza politica più significativa la componente più radicale e di destra, con delle possibili ricadute sul piano della stabilità politica. Insieme le liste Alleanza per la Sicilia-Musumeci Presidente-Fratelli d'Italia-Noi con Salvini e DiventeràBellissima esprimono quasi il $12 \%$ dei consensi, un quinto della maggioranza dei seggi necessari a garantire un governo stabile.

La tendenza alla "polarizzazione" del sistema politico siciliano investe anche i due blocchi che incarnano il ruolo dell'opposizione. Da un lato il centro-sinistra, attraversato dal risultato deludente della componente moderata della coalizione, che ha visto ridotta la sua rappresen-

${ }^{51}$ Effetto normalizzazione del M5s che emerge anche dai dati sui campioni delle preferenze nelle elezioni regionali del 2017 - vale a dire quei candidati che hanno superato le 10 mila preferenze - che, oltre ad evidenziare la trasversalità politica di questi candidati, sottolinea la presenza di ben 5 campioni delle preferenze del M5s. 
tanza (in numeri di seggi) e dall' 'ingresso in Assemblea della sinistra radicale - rappresentata dalla lista Cento Passi per la Sicilia -, pur mantenendo complessivamente gli stessi consensi del 2012, dunque, con uno spostamento a sinistra del punto di equilibrio potenziale dell'intera coalizione. Dall'altro lato il M5s che, oltre i dati sulla ri-conferma nelle elezioni regionali, dimostra sempre maggiore radicamento nell'Isola. La Sicilia è la regione con più sindaci del M5S rispetto al resto dell'Italia (8 su 45 comuni amministrati nel complesso). La strutturazione nel sistema politico siciliano del movimento e la consapevolezza di potere costituire una valida alternativa politica ai due blocchi (centrodestra e centrosinistra) spinge verso una radicalizzazione sui temi e sulle posizioni - come dimostrato anche dall'impostazione strategico-comunicativa -, dunque, ben lontane dalle esperienze di collaborazione sperimentate all'inizio della XVI legislatura con il Governo Crocetta.

\section{Riferimenti Bibliografici}

ATLANTE POlitico 69 (2017), «Elezioni Regione Sicilia», a cura di R. BIORCIO e F. BORDIGNON, Demos \& Pi, ottobre 2017.

AZzOLINA, L. (2013), «Sicilia, anno zero? I primi passi del nuovo governo regionale», il Mulino, 4, pp. 585-592.

BIORCIO, R., e F. BORDignON (2017), Regionali Sicilia, testa a testa centrodestra-M5s. La sinistra spaccata è molto indietro, Repubblica.it Palermo, 20 ottobre.

BOLGHERINI, S., e S. GRIMALDI (a cura di) (2015), Tripolarismo e destrutturazione. Le elezioni regionali del 2015, Bologna, il Mulino - Misure-Materiali di ricerca Istituto Cattaneo.

BOLGHERINI, S., e F. MUSELLA (2006), «Le primarie in Italia: ancora e soltanto personalizzazione della politica», Quaderni dell'Osservatorio elettorale, 55, pp. 219-239.

CACIAGLI, M., e P. SCARAMOZZINO (a cura di) (1983), Il voto di chi non vota, Milano, Edizioni Cominità.

CACiAgli, M., e A. Di VirgiLio (2005), Eleggere il sindaco. La nuova democrazia locale in Italia e in Europa, Torino, Utet.

CALISE, M. (2010), Il partito personale. I due corpi del leader, Roma-Bari, Laterza (1 ed. 2000).

CECCANTI, S. (2002), «La forma neoparlamentare di governo alla prova della dottrina e della prassi», Quaderni costituzionali, 1, pp. 107-126.

CERruto, M. (2008), «La rappresentanza politica regionale: Sicilia e Toscana in prospettiva comparata», Rivista Italiana di Scienza Politica, 1, pp. 55-87.

CERRUTO, M., e F. RANIOLO (2009), «Dal partito dominante alla coalizione dominante: le elezioni regionali in Sicilia (1947-2008)», Quaderni dell'Osservatorio elettorale, 61, pp. 4198.

COLlocA, P., e R. VignATI (2013), «Tra continuità e cambiamenti. Le elezioni regionali siciliane del 2012», Istituzioni del Federalismo, 1, pp. 265-301.

D’AMiCO, R. (1993), La "cultura elettorale" dei siciliani, in Far politica in Sicilia, a cura di M. MORISI, Milano, Feltrinelli.

DANDOY, R., e A.H. SCHAKEL (2013), Regional and national elections in Western Europe: territorially of the vote in thirteen countries, Palgrave Macmillan UK.

DiAmANTI, I. (2003), Bianco, rosso, verde... e azzurro. Mappe e colori dell'Italia politica, Bologna, Il Mulino.

EMANUELE, V., e B. MARINO (2016), «Follow the candidates, not the parties? Personal vote in a regional de-institutionalised party system», Regional and Federal Studies, 26 (4), pp. 531-554.

EMANUELE, V., e A. RigGiO (2017), «Trasformismo e adeguamento strategico: l'offerta politica in Sicilia», Cise, 14 ottobre 2017.

FERRAZZA, R. (2017), Sicilia, la galassia ex Dc è il secondo partito in regione, Il Sole24Ore, 7 novembre.

HiRSChMAn, A.O. (1970), Exit, Voice and Loyalty. Responses to Decline in Firms, Organizations, and States, Harvard, Harvard University Press. 
KenIG, O. (2008), «Democratization of Party Leadership Selection: Do wider Selectorates produce more competitive Contests?», Electoral Studies, 2, pp. 240-247.

LANZONE, M.E., e M. MORINI (2016), Dalle Regionali alle Regionali: un'analisi empirica delle primarie a Cinque Stelle, in Selezionare i presidenti. Le primarie regionali in Italia, a cura di M. DE LuCA e S. ROMBI, Novi Ligure, Epoké.

LANCELOT, A. (1968), L'anstenssionisme électoral en France, Parigi, A. Colin.

LEGNANTE, G. (2003), «La personalizzazione del voto. Come la vedono i parlamentari italiani», Quaderni dell'Osservatorio elettorale, 50, pp. 9-38.

Noelle-Neumann, E. (2002), La spirale del silenzio. Per una teoria dell'opinione pubblica, Roma, Meltemi.

RANIOLO, F. (2017), «Una Sicilia che sorprende», il Mulino, 13 novembre 2017.

REPORT \#OPENARS EDIZIONE FINALE (2017), monitoraggio aggiornato il 4 settembre 2017, a cura di A. Alu', F. GiaquinTA, C. LONGO e A. Stella, Analisi dell'attività parlamentare dei deputati regionali nel corso della XVI legislatura in base ai risultati di ricerca pubblicamente disponibili all'interno del portale web dell'Assemblea Regionale Siciliana, http://www.ars.sicilia.it/default.jsp.

REPORT \#CAMBIOGRUPPO, REPORT STATISTICO RELATIVO ALLE VARIAZIONI DEI GRUPPI PARLAMENTARI ARS (2016), monitoraggio aggiornato il 20 novembre 2016.

SAMPUGNARO, R., e F. MONTEMAGNO (2016), La campagna lunga ma anche cortissima. Le strategie dei candidati alla Presidenza della Regione Siciliana nel 2017, in Dall'Europa alla Sicilia. Elezioni e opinione pubblica nel 2017, a cura di V. EMANUELE e A. PAPARO, Roma, Cise, pp. 213-226.

VASSALLO, S. (2005), «Le elezioni regionali del 2005. Alternanze, personalizzazione e ricambio», Le Istituzioni del Federalismo, 6, pp. 987-1006.

VASSALlO, S., e G. BALDINI (2000), Sistemi di partito, forma di governo e politica di coalizione nelle Regioni italiane, in Elezioni, assemblee e governi regionali (1947-2000), a cura di G. BALDINI e S. VASSALlO, Le Istituzioni del Federalismo, 3-4.

VignAt, R. (2017), Regionali Sicilia: un'Isola dall'elettorato fluido, Bologna, Istituto Cattaneo. 